\title{
Two types of co-accretion scenarios for the origin of the Moon
}

\author{
Ryuji Morishima and Sei-ichiro Watanabe \\ Department of Earth and Planetary Sciences, Nagoya University, Nagoya 464-8602, Japan
}

(Received May 24, 2000; Revised October 19, 2000; Accepted December 19, 2000)

\begin{abstract}
Based on orbital calculations of Keplerian planetesimals incident on a planet with various initial orbital elements, we develop a numerical model which describes the accretional and dynamical evolution of planet-satellite systems in a swarm of planetesimals on heliocentric orbits with given spatial and velocity distributions. In the plane of orbital radius of the satellite vs. satellite/planet mass ratio, a satellite with some initial value moves quickly toward the balanced orbital radius, where accretion drag compensates with tidal repulsion, and then grows toward the equilibrium mass ratio. Using the model, we propose two types of co-accretion scenarios for the origin of the Moon, both of which satisfy the most fundamental dynamical constraints: the large angular momentum of the Earth-Moon system and the large Moon/Earth mass ratio. In the first scenario the Moon starts from a small embryo and grows in a swarm of planetesimals with low velocity dispersion and nonuniform spatial distribution, so that large spin angular momentum is supplied to the planet. Such a situation would be realized when the Earth grows up rapidly before dissipation of the solar nebula. Second one considers co-accretion after a giant impact during Earth accretion, which produces enough angular momentum as large as that of the present Earth-Moon system as well as a lunar-sized satellite. In this case, solar nebula would have already dissipated and random velocities of incident planetesimals are rather high, so that the Earth grows slowly. We find that the total angular momentum decreases by 5-25\% during this co-accretion stage.
\end{abstract}

\section{Introduction}

The origin of the Moon has been controversial for many years, but it has not been clarified yet (Boss and Peale, 1986; Ida et al., 1997). The lunar formation scenario must satisfy the following constraints: the large mass ratio of the Moon $\left(\mu_{\mathrm{M}}=M_{\mathrm{M}} / M_{\mathrm{E}}=1.23 \times 10^{-2}\right.$, where $M_{\mathrm{M}}$ and $M_{\mathrm{E}}$ denote the masses of the Moon and the Earth), the anomalously large angular momentum of the Earth-Moon system $\left(L_{\mathrm{EM}}=\right.$ $\left.3.46 \times 10^{41} \mathrm{~g} \mathrm{~cm}^{2} \mathrm{~s}^{-1}\right)$, the large obliquity of the Earth $(0.409$ $\mathrm{rad})$, the small orbital eccentricity of the Earth-Moon system around the Sun (0.017), and some geochemical constraints especially the depletion of iron and volatile elements in the bulk composition of the Moon. However, no hypotheses can satisfy all of the constraints yet.

Classifying the lunar-formation hypotheses by the direct origin of a lunar embryo, impact and capture are considerable. The giant-impact hypothesis is very popular (Benz et al., 1986, 1987, 1989; Cameron and Benz, 1991; Cameron, 1997; Ida et al., 1997), which is mostly favorable from the dynamical and geochemical constraints. In the capture hypotheses, capture by gas drag (Nakazawa et al., 1983), by mutual tidal interactions (Singer and Bandermann, 1970), and by collisions between planetesimals in the Hill sphere of the Earth (Ruskol, 1960) have been proposed. The capture hypotheses, however, have been considered not to be plausible from the geochemical constraints. Note that co-accretion is not one that specifies the direct origin of the lunar em-

Copy right (C) The Society of Geomagnetism and Earth, Planetary and Space Sciences (SGEPSS); The Seismological Society of Japan; The Volcanological Society of Japan; The Geodetic Society of Japan; The Japanese Society for Planetary Sciences. bryo. Once a satellite formed around the planet by some mechanism, it would grow together with the planet under accretion of planetesimals. The co-accretion stage inevitably exists whether its period is long or short, so that one must investigate not only the direct origin but also the dynamical evolution of the co-accretion stage.

In the co-accretion stage, accumulating planetesimals supply mass and angular momentum to the planet and the satellite. The spin angular momentum of the planet is then transferred to the orbital angular momentum of the satellite by their tidal interaction. In the present paper, we propose a new method for calculating accretional and dynamical evolutions (i.e., semimajor axis of the satellite) of planet-satellite systems in a swarm of planetesimals on heliocentric orbits with given spatial and velocity distributions.

In their pioneer works, Harris and Kaula (1975) (hereafter HK75) and Harris (1978) calculated evolution of the Moon/Earth mass ratio and the semimajor axis of the Moon in a swarm of planetesimals using the particle-in-a-box approximation. HK75 showed that under the balance between accretion drag and tidal repulsion, the semimajor axis of the proto-Moon is kept about 10 times of proto-Earth radius in the course of co-accretion. They also found a lunar progenitor would grow from an initial mass of $10^{-4}$ of the proto-Earth to the present lunar mass while the Earth itself grows from $0.1 M_{\mathrm{E}}$ to the present mass. The rapid growth of the Moon relative to the Earth growth is because their adopted random velocities are so large that the ratio of collisional cross section of the Earth to that of the Moon is determined mainly by the ratio of their geometrical cross sections.

Harris (1978) extended the model of HK75 including the 
effect of planetary-ring materials produced by mutual collisions of planetesimals in the Hill sphere of the planet. However, these contributions are considered to be rather small, since the mass of the planetary ring produced by such a mechanism is generally much smaller than the present lunar mass (Stevenson et al., 1986). Hence, the evolution of mass and angular momentum of the Earth-Moon system should be determined mainly by direct collisions of planetesimals to the Earth and the Moon. A fatal problem of the model of HK75 is that the final spin angular momentum of the Earth supplied by planetesimals with large relative-random velocities as adopted by HK75 is much smaller than $1 L_{\mathrm{EM}}$ (Ida and Nakazawa, 1990; Lissauer and Kary, 1991; Dones and Tremaine, 1993a).

Two mechanisms have been proposed to explain the large angular momentum of the Earth-Moon system. One is continuous accretion of planetesimals with spatially nonuniform distributions (Ohtsuki and Ida, 1998; hereafter OI98). When a clear gap of planetesimals in the vicinity of a protoplanet is formed due to the balance between gravitational scattering of the protoplanet and gas drag of the solar nebula (Tanaka and Ida, 1997, 1999), only planetesimals of lower Jacobi energies can collide to the planet and supply net prograde angular momentum, which fairly explain the angular momentum of the Earth-Moon system (OI98). The other invokes the giant impact hypothesis. An oblique collision between a Mars-sized protoplanet and the proto-Earth brings forth the rapid spin of the proto-Earth as well as orbiting debris from which the Moon is made. If the total mass of the proto-Earth and the impactor is larger than say $0.5 M_{\mathrm{E}}$, formed system can have angular momentum larger than $1 L_{\mathrm{EM}}$ (Cameron and Canup, 1998a).

The plausible mechanism of angular momentum supplement depends on a adopted formation scenario of the terrestrial planets. The recent studies of planetary accretion reveal that a small number of protoplanets with masses of $0.1 M_{\mathrm{E}}$ are formed in the terrestrial zone by runaway growth (Wetherill and Stewart, 1993; Kokubo and Ida, 1996, 1998). However, the subsequent stage of planetary formation is kept unclear.

One possible case is that each protoplanet is isolated from other protoplanets and grows rapidly by sweeping up a large number of planetesimals in the course of its radial migration (Tanaka and Ida, 1999) due to tidal interaction between the solar nebula and the protoplanet (e.g., Ward, 1997; and references therein). In this case, protoplanets may have circular orbits due to the dynamical friction exerted by the surrounding planetesimals (or by the solar nebula).

The other case is that each protoplanet grows rather slowly, so that the solar nebula is disappeared before the completion of the growth. Then the orbital crossings due to mutual interactions may bring forth giant impacts. Recent $N$-body simulations support the picture, but it is hard to explain the present small orbital eccentricities of the terrestrial planets (Chambers and Wetherill, 1998; Ito and Tanikawa, 1999; Agnor et al., 1999).

Corresponding to the two pictures of planetary accretion, we propose two types of co-accretion scenarios for the origin of the Moon: co-accretion from small embryos in the solar nebula and co-accretion after the later-stage giant impact in vacuum.
In the former scenario, a small lunar embryo is assumed to be formed by some mechanism such as capture due to gas drag or an oblique impact when the mass of the protoEarth is much smaller than $1 M_{\mathrm{E}}$. We examine whether such a small embryo can grow up to the Moon in the swarm of planetesimals with nonuniform spatial distribution and lower random velocities, both of which guarantee the large angular momentum of the Earth-Moon system. It should be noted that if the Moon gains most of the mass in the co-accretion, it might be hard to explain the bulk composition of the Moon. But it is not clear from the chemical constraints how much the mass is allowed to accumulate onto the Moon in the coaccretion. So, first we will clarify dynamical constraints on the co-accretion process (period, velocity dispersion of incident planetesimals, etc.) in the present paper.

The latter scenario invokes co-accretion after the Moonforming giant impact. The debris of the impact-generated disk would accrete to a single satellite within a year (Ida $e t$ al., 1997). Judging from the proportion of incorporation of the disk into the satellite $(\lesssim 0.4)$, the disk masses formed by impacts with angular momentum $1 L_{\mathrm{EM}}$ and total mass $1 M_{\mathrm{E}}$ (e.g., Cameron and Benz, 1991) are found to be generally insufficient to account for the lunar mass. However, Cameron and Canup (1998a, b) showed that impacts with angular momentum about $1 L_{\mathrm{EM}}$ and total mass roughly $\frac{2}{3} M_{\mathrm{E}}$ can produce the disks massive enough to form the Moon. Thus the giant impact during Earth accretion is plausible and we should investigate co-accretion afterwards to clarify the change of mass ratio of the Moon as well as angular momentum of the Earth-Moon system due to accretion of rather high-speed planetesimals in vacuum.

In Section 2, we present methods of calculating accretion rates of mass and angular momentum supplied to the planet and the satellite from planetesimals with given spatial and velocity distributions. The results are shown in Section 3 for wide ranges of parameters. In Section 4, we apply the results to the two types of lunar formation scenarios and discuss the plausibility. In Section 5, we compare our results for co-accretion after the giant impact with results of simulations of satellite-forming impacts, and constrain the physical parameter of the Moon-forming impact. In this section, we also discuss the effects of imperfect coalescence between the satellite and planetesimals, gas drag on the satellite, and stochastic impacts. Finally, our summary is presented in Section 6.

\section{Methods}

\subsection{Basic equations and assumptions}

We consider a planet-satellite system rotating around the Sun in a swarm of planetesimals. In order to examine accretional and dynamical evolution of the planet-satellite system, we numerically evaluate mass and planetocentric angular momentum fluxes supplied to the satellite as well as to the planet by numerous orbital calculations of incident planetesimals with different orbital elements.

In order to calculate orbital motion of massless planetesimals, we adopt the following assumptions:

A1 The instantaneous eccentricities and inclinations of planetesimals are much smaller than unity. 
A2 The center of mass of the planet-satellite system has a circular orbit around the Sun.

We adopt a rotating local Cartesian coordinate with the origin at the center of the mass of the planet-satellite system, $x$ taken along the radial direction, $y$ along the azimuthal direction, and $z$ along the vertical direction. The equations of motion of the satellite are given as (e.g., Nakazawa and Ida, 1988)

$$
\left\{\begin{array}{l}
\ddot{x}_{\mathrm{s}}=+2 \dot{y}_{\mathrm{s}}+3 x_{\mathrm{s}}-\frac{3 x_{\mathrm{s}}}{\left|\boldsymbol{x}_{\mathrm{s}}\right|^{3}}, \\
\ddot{y}_{\mathrm{s}}=-2 \dot{x}_{\mathrm{s}}-\frac{3 y_{\mathrm{s}}}{\left|\boldsymbol{x}_{\mathrm{s}}\right|^{3}}, \\
\ddot{z_{\mathrm{s}}}=-z_{\mathrm{s}}-\frac{3 z_{\mathrm{s}}}{\left|\boldsymbol{x}_{\mathrm{s}}\right|^{3}},
\end{array}\right.
$$

where $\boldsymbol{x}_{\mathrm{s}}=\left(x_{\mathrm{s}}, y_{\mathrm{s}}, z_{\mathrm{s}}\right)$ is the position of the satellite relative to the planet. The equations of motion of a planetesimal are given by

$$
\left\{\begin{aligned}
& \ddot{x}=+2 \dot{y}+3 x-3 \frac{1}{1+\mu_{\mathrm{s}}} \frac{x}{|\boldsymbol{x}|^{3}}-3 \frac{\mu_{\mathrm{s}}}{1+\mu_{\mathrm{s}}} \frac{x_{\mathrm{s}}}{\left|\boldsymbol{x}_{\mathrm{s}}\right|^{3}} \\
&-3 \frac{\mu_{\mathrm{s}}}{1+\mu_{\mathrm{s}}} \frac{x-x_{\mathrm{s}}}{\left|\boldsymbol{x}-\boldsymbol{x}_{\mathrm{s}}\right|^{3}}, \\
& \ddot{y}=-2 \dot{x} \quad-3 \frac{1}{1+\mu_{\mathrm{s}}} \frac{y}{|\boldsymbol{x}|^{3}}-3 \frac{\mu_{\mathrm{s}}}{1+\mu_{\mathrm{s}}} \frac{y_{\mathrm{s}}}{\left|\boldsymbol{x}_{\mathrm{s}}\right|^{3}}-3 \frac{\mu_{\mathrm{s}}}{1+\mu_{\mathrm{s}}} \frac{y-y_{\mathrm{s}}}{\left|\boldsymbol{x}-\boldsymbol{x}_{\mathrm{s}}\right|^{3}}, \\
& \ddot{z}=\quad-z-3 \frac{1}{1+\mu_{\mathrm{s}}} \frac{z}{|\boldsymbol{x}|^{3}}-3 \frac{\mu_{\mathrm{s}}}{1+\mu_{\mathrm{s}}} \frac{z_{\mathrm{s}}}{\left|\boldsymbol{x}_{\mathrm{s}}\right|^{3}} \\
&-3 \frac{\mu_{\mathrm{s}}}{1+\mu_{\mathrm{s}}} \frac{z-z_{\mathrm{s}}}{\left|\boldsymbol{x}-\boldsymbol{x}_{\mathrm{s}}\right|^{3}},
\end{aligned}\right.
$$

where $\boldsymbol{x}=(x, y, z)$ is the position of the planetesimal relative to the planet. Further $\mu_{\mathrm{s}}$ is the mass ratio of the satellite given by $\mu_{\mathrm{s}}=M_{\mathrm{s}} / M_{\mathrm{p}}$, where $M_{\mathrm{p}}$ and $M_{\mathrm{s}}$ are the masses of the planet and the satellite, respectively. Equations (1) and (2) are written in non-dimensional forms with time normalized by the inverse of the Keplerian angular velocity $\Omega_{\mathrm{K}}^{-1}$ of the center of mass of the planet-satellite system and distance by the Hill radius $R_{\mathrm{H}}$ defined by

$$
R_{\mathrm{H}}=A_{\mathrm{p}} h=A_{\mathrm{p}}\left(\frac{M_{\mathrm{p}}+M_{\mathrm{s}}}{3 M_{*}}\right)^{1 / 3},
$$

where $A_{\mathrm{p}}$ is the heliocentric distance of the center of mass. Here we also introduced the reduced Hill radius $h=R_{\mathrm{H}} / A_{\mathrm{p}}$.

When the distance between the planet-satellite system and the planetesimal is large enough, the analytic solution of Eq. (2) reduces to simple Keplerian motion. We adopt the following scaled orbital elements:

$$
b=\frac{A-A_{\mathrm{p}}}{R_{\mathrm{H}}}, \quad e=\frac{e^{*}}{h}, \quad i=\frac{i^{*}}{h},
$$

where $A, e^{*}$, and $i^{*}$ are, respectively, the semimajor axis, eccentricity, and inclination of the planetesimal in ordinary use.

To simplify the problem, we further assume that

A3 The orbital plane of the satellite is coplanar with that of the planet-satellite system around the Sun.

A4 The planetocentric orbit of the satellite is circular and prograde.
Then the free orbital elements of the satellite are only two: the planetocentric distance of the satellite $a_{\mathrm{s}}\left(=A_{\mathrm{s}} / R_{\mathrm{H}}\right.$, where $A_{\mathrm{s}}$ denotes the dimensional distance) and the initial phase angle $\varphi_{\mathrm{s}}$, which determines the position of the satellite.

We consider that a planetesimal collides with the planet or the satellite if the relative distances become smaller than each physical radius. The physical radius of the planet normalized by $R_{\mathrm{H}}$ is given by

$$
r_{\mathrm{p}}=0.005\left(\frac{\rho_{\mathrm{p}}}{3.0 \mathrm{~g} \mathrm{~cm}^{-3}}\right)^{-1 / 3}\left(\frac{A_{\mathrm{p}}}{1 \mathrm{AU}}\right)^{-1},
$$

where $\rho_{\mathrm{p}}$ denotes the density of the planet. In the following orbital calculations, we adopt the radius of the planet $r_{\mathrm{p}}$ to be 0.005 . The radius of the satellite is given by $r_{\mathrm{s}}=$ $\left(\rho_{\mathrm{p}} / \rho_{\mathrm{s}}\right)^{1 / 3}\left(M_{\mathrm{s}} / M_{\mathrm{p}}\right)^{1 / 3} r_{\mathrm{p}}$, where $\rho_{\mathrm{s}}$ denotes the density of the satellite.

Morishima and Watanabe (1996) obtained the growth rates of the planet and the satellite by numerical integrations of Eq. (2) in the case for $e=i=0$. They fully took into account the gravity of the satellite since mass ratios of the satellite were so large $\left(0.05 \leq \mu_{\mathrm{s}} \leq 1\right)$ in their calculations. In general, there are 8 parameters needed for uniquely specifying the orbit of a planetesimal $\left(b, e, i, \tau, \omega, a_{\mathrm{s}}, r_{\mathrm{s}}\right.$, and $\varphi_{\mathrm{s}}$; where $\tau$ and $\omega$ are the longitude of periheiron and the longitude of ascending node of planetesimals, respectively). In order to reduce the number of parameters, we here examine the condition that the satellite gravity makes negligible contribution to the orbital motion of planetesimals:

A5 The relative collision velocities of planetesimals to the satellite are much larger than the escape velocity of the satellite $v_{\mathrm{esc}, \mathrm{s}}=\left(6 \mu_{\mathrm{s}} / r_{\mathrm{s}}\right)^{1 / 2}\left(=\left(2 G M_{\mathrm{s}} / R_{\mathrm{s}}\right)^{1 / 2} / R_{\mathrm{H}} \Omega_{\mathrm{K}}\right.$, where $G$ is the gravity constant).

Then neglecting the relative velocities of planetesimals with respect to the heliocentric orbit of the planet, we obtain the sufficient condition for neglecting the satellite gravity as

$$
a_{\mathrm{s}} \ll \frac{3}{2} \frac{r_{\mathrm{s}}}{\mu_{\mathrm{s}}} .
$$

For a lunar-sized satellite $\left(\mu_{\mathrm{s}} \simeq 0.012, r_{\mathrm{s}} \simeq 1.2 \times 10^{-3}\right)$, we obtain $a_{\mathrm{s}} \ll 0.15 \simeq 30 r_{\mathrm{p}}$. In most of the following we consider smaller $\mu_{\mathrm{s}}$ or $a_{\mathrm{s}}$, so that we can safely neglect the effect of satellite gravity in calculating planetesimal orbits. Thus, we put $\mu_{\mathrm{s}}=0$ in Eq. (2) in the following orbital calculations. Even for the cases with larger satellites, we can fairly estimate the growth rate of the satellite by simply multiplying the gravitational focusing factor $\alpha=1+\left(v_{\mathrm{esc}, \mathrm{s}} / v_{\mathrm{r}, \mathrm{s}}\right)^{2}$ of the satellite, where $v_{\mathrm{r}, \mathrm{s}}$ denotes the averaged relative velocity of planetesimals to the satellite.

The collision rate $P_{\mathrm{p}}(e, i)$ of planetesimals to the planet is given, from orbital calculations, by (Ida and Nakazawa, 1989)

$$
P_{\mathrm{p}}(e, i)=\int \frac{3}{2}|b| \sigma_{\mathrm{d}}(b) p_{\mathrm{col}, \mathrm{p}}(e, i, b, \tau, \omega) \frac{d \tau d \omega}{(2 \pi)^{2}} d b,
$$

where $p_{\text {col,p }}$ is unity for collision orbits and zero otherwise, and $\sigma_{\mathrm{d}}(b)$ is the nondimensional surface density of planetesimals at $b$. Here we neglect the effect of the satellite as a hindrance. The mean specific spin angular momentum supplied to the planet normalized by the specific 


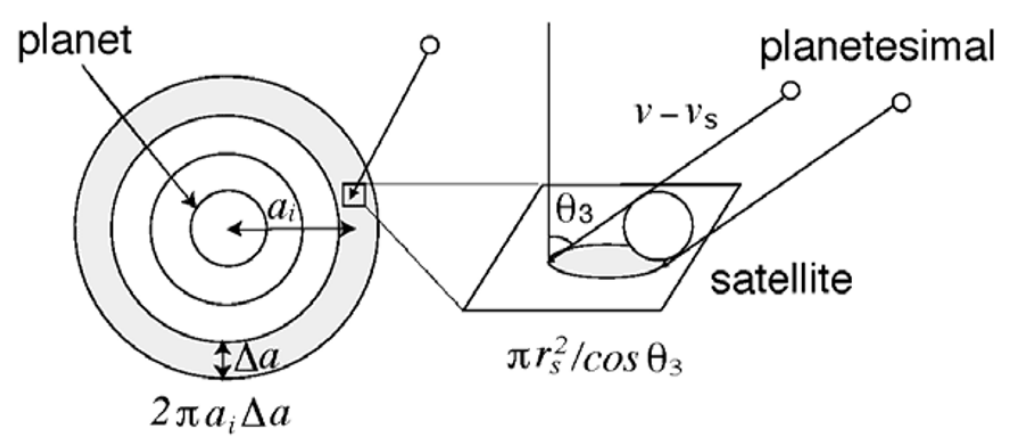

Fig. 1. Schematic illustration of oblique penetration of a planetesimal through an $i$ th planetocentric annulus with radius $a_{i}$ and width $\Delta$ a. Here $\theta_{3}$ is the angle between relative-velocity vector of the planetesimal to the satellite and normal direction of the satellite's orbital plane, and $r_{\mathrm{s}}$ is radius of the satellite.

orbital angular momentum of a grazing satellite $\left(3 r_{\mathrm{p}}\right)^{1 / 2}$ $\left[=\left(G M_{\mathrm{p}} R_{\mathrm{p}}\right)^{1 / 2} /\left(R_{\mathrm{H}}^{2} \Omega_{\mathrm{K}}\right)\right]$ is given by

$$
\begin{aligned}
J_{\mathrm{p}}(e, i)= & \left\langle\frac{\ell_{z}}{\left(3 r_{\mathrm{p}}\right)^{1 / 2}}\right\rangle \\
= & \frac{1}{P_{\mathrm{p}}} \int \frac{3}{2}|b| \sigma_{\mathrm{d}}(b) \frac{\ell_{z}(e, i, b, \tau, \omega)}{\sqrt{3 r_{\mathrm{p}}}} \\
& \cdot p_{\mathrm{col}, \mathrm{p}}(e, i, b, \tau, \omega) \frac{d \tau d \omega}{(2 \pi)^{2}} d b .
\end{aligned}
$$

In the above $\ell_{z}$ is the $z$-component of the specific planetocentric angular momentum given by a colliding planetesimal:

$$
\ell_{z}(e, i, b, \tau, \omega)=r \dot{\varphi}+r^{2},
$$

where we use a planetocentric cylindrical coordinate for convenient sake; the position and the velocity of the planetesimal at the collision are given by $(r, \varphi, z)$ and $(\dot{r}, \dot{\varphi}, \dot{z})$, respectively.

\subsection{Evaluation of the accretion rate of mass and specific angular momentum supplied to the satellite}

For a satellite at planetocentric distance $a_{\mathrm{s}}$, the collision rate $P_{\mathrm{s}}\left(e, i, a_{s}, r_{\mathrm{s}}\right)$ and the mean specific planetocentric angular momentum $J_{\mathrm{s}}\left(e, i, a_{\mathrm{s}}\right)$ normalized by the satellite orbital angular momentum $\ell_{\mathrm{o}}\left[=\left(3 a_{\mathrm{s}}\right)^{1 / 2}\right]$ are, respectively, given by

$$
\begin{gathered}
P_{\mathrm{s}}\left(e, i, a_{\mathrm{s}}, r_{\mathrm{s}}\right)=\int \frac{3}{2}|b| \sigma_{\mathrm{d}}(b) p_{\mathrm{col}, \mathrm{s}}\left(e, i, b, \tau, \omega, a_{\mathrm{s}}, r_{\mathrm{s}}, \varphi_{\mathrm{s}}\right) \\
\cdot \frac{d \varphi_{\mathrm{s}} d \tau d \omega}{(2 \pi)^{3}} d b, \\
J_{\mathrm{s}}\left(e, i, a_{\mathrm{s}}\right)=\left\langle\frac{\ell_{z}}{\ell_{\mathrm{o}}}\right\rangle=\frac{1}{P_{\mathrm{s}}} \int \frac{3}{2}|b| \sigma_{\mathrm{d}}(b) \frac{\ell_{z}}{\ell_{\mathrm{o}}}(e, i, b, \tau, \omega) \\
\cdot p_{\mathrm{col}, \mathrm{s}}\left(e, i, b, \tau, \omega, a_{\mathrm{s}}, r_{\mathrm{s}}, \varphi_{\mathrm{s}}\right) \\
\frac{d \varphi_{\mathrm{s}} d \tau d \omega}{(2 \pi)^{3}} d b,
\end{gathered}
$$

where $p_{\text {col,s }}$ is unity for collision orbits to the satellite and zero otherwise. It should be noted that if $J_{\mathrm{s}}>1$, the specific angular momentum of the satellite increases and its orbit expands.

Since we neglect satellite gravity, the orbits of planetesimals are not affected by the position of the satellite, so that only one orbital calculation is needed for each planetesimal of given orbital elements. Further we safely expect the principle of equal a priori probabilities in the orbital phase of the satellite. Then, instead of counting direct collisions to each satellite, we merely count number of penetrating orbits through concentric annuli with the center at the planet and width $\Delta a$ (see Fig. 1). When a planetesimal with given orbital elements penetrates the $i$ th annulus, the collision probability between the satellite therein and the planetesimal is given by the ratio of the projection area of the satellite body to the total area of the annulus:

$$
\begin{aligned}
& p_{\mathrm{col}, 3 \mathrm{D}}\left(e, i, b, \tau, \omega, a_{i}, r_{\mathrm{s}}\right) \\
& =\frac{1}{2 \pi a_{i} \Delta a} \\
& \cdot \int_{a_{i}-\Delta a / 2}^{a_{i}+\Delta a / 2} \int_{0}^{2 \pi} p_{\mathrm{col}, \mathrm{s}}\left(e, i, b, \tau, \omega, a_{s}, r_{\mathrm{s}}, \varphi_{\mathrm{s}}\right) a_{\mathrm{s}} d \varphi_{\mathrm{s}} d a_{\mathrm{s}} \\
& = \begin{cases}\frac{g_{3} r_{\mathrm{s}}^{2}}{2 a_{i} \Delta a} & \text { (for penetrating orbits) } \\
0 & \text { (otherwise) }\end{cases}
\end{aligned}
$$

where $a_{i}$ is the mean radius of the $i$ th annulus. In the above, $g_{3}$ denotes the enlargement factor for oblique penetration given by

$$
g_{3} \simeq \min \left(\frac{1}{\cos \theta_{3}}, \frac{3 a_{\mathrm{i}}}{r_{\mathrm{s}}}\right) .
$$

Here $\theta_{3}$ is the angle between the incident direction of the planetesimal in the comoving system for the satellite and normal direction of the orbital plane of the satellite given by

$$
\tan \theta_{3}=\left|\frac{\sqrt{\left(r \dot{\varphi}-r \omega_{\mathrm{s}}\right)^{2}+\dot{r}^{2}+\dot{z}^{2}}}{\dot{z}}\right|,
$$

where $\omega_{\mathrm{s}}$ denotes the angular velocity of the satellite given by

$$
\omega_{\mathrm{s}}=\left(\frac{G M_{\mathrm{p}}}{A_{s}^{3}}\right)^{1 / 2} \Omega_{\mathrm{K}}^{-1}=\left(\frac{3}{a_{s}^{3}}\right)^{1 / 2} .
$$

The upper limit of $g_{3}$ in Eq. (13) is derived in Appendix A. We find that $1 / \cos \theta_{3}$ scarcely exceeds the upper limit $3 a_{\mathrm{i}} / r_{\mathrm{s}}$, when $i \gtrsim 0.01$, so that $g_{3}$ is almost independent of $r_{\mathrm{s}}$. Thus once we obtain the collision rate for a certain physical radius of the satellite as a standard case, we can estimate the value 


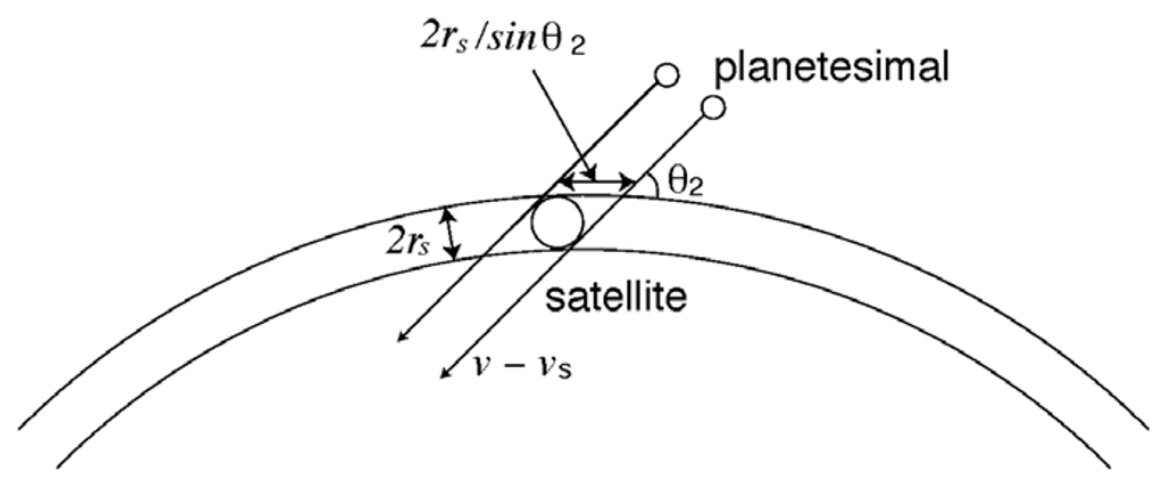

Fig. 2. Same as Fig. 1 but for the two-dimensional case $(i=0)$. Here $\theta_{2}$ is the angle between relative-velocity vector of the planetesimal to the satellite and direction of the satellite's orbital motion.

for any other radius by multiplying the square of the ratio of the radius to the standard value except extremely low $i$ cases.

We find that the results have little dependence on $\Delta a$ if $\Delta a$ is smaller than 0.01. So we fix $\Delta a=0.01$ in the following.

In order to know the behavior of $P_{\mathrm{S}}$ in the cases of low inclination case, we also calculate $P_{\mathrm{s}}$ and $J_{\mathrm{s}}$ for purely twodimensional cases. When a planetesimal orbit crosses the satellite orbit, the collision probability is given by the ratio of the satellite diameter to the length of the orbital circumference (see Fig. 2):

$$
\begin{aligned}
& p_{\mathrm{col}, 2 \mathrm{D}}\left(e, i=0, b, \tau, a_{\mathrm{s}}, r_{\mathrm{s}}\right) \\
& =\int_{0}^{2 \pi} p_{\mathrm{col}, \mathrm{s}}\left(e, i=0, b, \tau, a_{s}, r_{\mathrm{s}}, \varphi_{\mathrm{s}}\right) \frac{d \varphi_{\mathrm{s}}}{2 \pi} \\
& = \begin{cases}\frac{g_{2} r_{\mathrm{s}}}{\pi a_{\mathrm{s}}} & \text { (for crossing orbits), } \\
0 & \text { (otherwise) } .\end{cases}
\end{aligned}
$$

Here $g_{2}$ is the enlargement factor for oblique crossing given by

$$
g_{2}=\min \left(\frac{1}{\sin \theta_{2}}, 4 \sqrt{\frac{a_{\mathrm{s}}}{2 r_{\mathrm{s}}}}\right),
$$

where $\theta_{2}$ is the angle between the relative-velocity vector of the planetesimal to the satellite and the velocity vector of the satellite given by

$$
\cos \theta_{2}=\left|\frac{a_{\mathrm{s}} \dot{\varphi}-a_{\mathrm{s}} \omega_{\mathrm{s}}}{\sqrt{\left(a_{\mathrm{s}} \dot{\varphi}-a_{\mathrm{s}} \omega_{\mathrm{s}}\right)^{2}+\dot{r}^{2}}}\right| .
$$

The upper limit of $g_{2}$ in Eq. (17) can be derived, considering a parabolic tangential orbit with the satellite orbit. We find that orbits with $1 / \sin \theta_{2}>4 \sqrt{a_{\mathrm{s}} / 2 r_{\mathrm{s}}}$ are very rare, so that $g_{2}$ is almost independent of $r_{\mathrm{s}}$.

In order to obtain $P_{\mathrm{s}}$ and $J_{\mathrm{s}}$, we numerically integrate Eq. (2) using a fourth-order time-step variable Runge-Kutta method. An orbital integration of a planetesimal with given orbital elements is continued until the planetesimal collides with the planet or goes far away from the planet-satellite system.

According to OI98, we modeled spatial distributions of planetesimals as follows. For an uniform case, nondimensional surface number density $\sigma_{\mathrm{d}}(b)$ is unity for all $b$. On the other hand, for nonuniform cases, planetesimals only exist in the range of $E \leq E_{\max }$, where $E$ denotes the Jacobi integral given by (e.g., Nakazawa and Ida, 1988)

$$
E=\frac{1}{2}\left(e^{2}+i^{2}\right)-\frac{3}{8} b^{2}+\frac{9}{2},
$$

and $E_{\max }$ denotes the maximum value of $E$. Thus $\sigma_{\mathrm{d}}(b)$ for nonuniform cases is given by

$\sigma_{\mathrm{d}}(b)=\left\{\begin{array}{l}0 \text { for }|b|<\left[4\left(e^{2}+i^{2}\right) / 3+12-8 E_{\max } / 3\right]^{1 / 2}, \\ 1 \text { for }|b| \geq\left[4\left(e^{2}+i^{2}\right) / 3+12-8 E_{\max } / 3\right]^{1 / 2}\end{array}\right.$

We perform calculations for the uniform case (OI98 calls it the case of $E_{\max }=\infty$ ) and two nonuniform cases with $E_{\max }=1.5$ and 2.0. In the case of $E_{\max } \sim 1.5$, the final spin angular momentum of the planet was found to be as large as the present total angular momentum of the Earth-Moon system (OI98). We assume that planetesimals are distributed uniformly in the phase space $(\tau, \omega)$ for all the cases.

\section{Results}

\subsection{Collision rate of planetesimals to the satellite}

Using the methods described in Subsection 2.2, we numerically calculate the collision rate of planetesimals to the satellite from the data of more than $10^{4}$ orbits penetrating each annulus of Fig. 1. Though we only show the results for the case of $r_{\mathrm{s}}=0.001$, which corresponds to $\mu_{\mathrm{s}}=0.01$ with $\rho_{\mathrm{p}}=\rho_{\mathrm{s}}$, one can easily obtain $P_{\mathrm{s}}$ for an arbitrary radius by multiplying the ratio of geometrical cross sections.

Using Eq. (10) we first examine the collision rate of the satellite in a planetesimal disk with uniform spatial distribution. Figure 3 shows $P_{\mathrm{s}}$ as a function of $a_{\mathrm{s}}$ for various values of $e$ with fixing the ratio $i / e$ to be 0.5 . We find the following properties: (1) in all cases $P_{\mathrm{s}}$ decrease with the increase of $a_{\mathrm{s}}$ and $e$, and the simple approximate relation $P_{\mathrm{s}} \propto a_{\mathrm{s}}^{-1}$ is valid for $1 \leq e \leq 8$; (2) $P_{\mathrm{s}}(e=i=0)$ is almost independent of $a_{\mathrm{s}}$ for $a_{\mathrm{s}} \lesssim 0.05$ and is approximated as a power law $P_{\mathrm{s}}(e=i=0) \propto a_{\mathrm{s}}^{-1}$ for larger $a_{\mathrm{s}}$, and similar $a_{\mathrm{s}}$-dependencies of $P_{\mathrm{s}}$ can be seen for $e \lesssim 1.0$.

Property (1) is explained by using a semi-analytical form of collision rate $P_{\mathrm{s}, 2 \mathrm{~B}}$, which is practically valid for $e, i \gtrsim$ 1. This approximate form is basically after HK75 but the numerical factor is determined by the 2-body approximation (e.g., Greenzweig and Lissauer, 1990): 


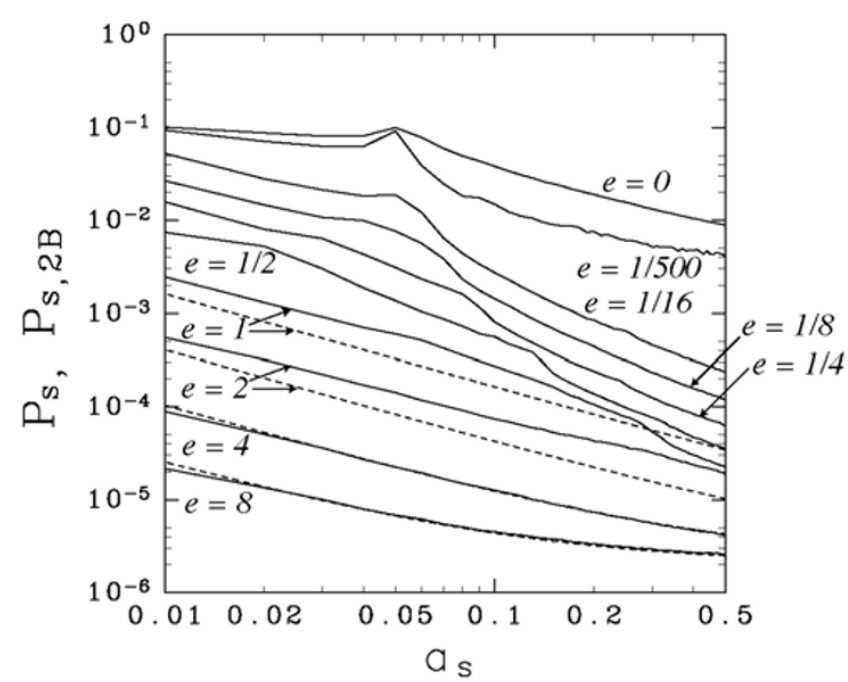

Fig. 3. Collision rate versus planetocentric distance of the satellite $\left(\mu_{\mathrm{s}}=0.01\right)$ in the case of uniform spatial distribution of planetesimals with various $e(0 \leq e \leq 8)$ with fixing $i / e=0.5$. The solid curves denote numerical solutions and the dashed curves in the case of $e \geq 1.0$ denote analytic solutions given by Eq. (20).

$$
\begin{aligned}
P_{\mathrm{s}, 2 \mathrm{~B}}\left(e, i, a_{\mathrm{s}}, r_{\mathrm{s}}\right)= & \left(\frac{2}{\pi}\right)^{2}[\boldsymbol{E}(k) \boldsymbol{K}(k)]^{1 / 2} \pi r_{\mathrm{s}}^{2} \\
& \cdot\left(1+\frac{6}{a_{\mathrm{s}} v_{\mathrm{r}, \mathrm{p}}^{2}}\right) \frac{v_{\mathrm{r}, \mathrm{p}}}{2 i},
\end{aligned}
$$

where $v_{\mathrm{r}, \mathrm{p}}$ is the random velocity of planetesimals given by

$$
v_{\mathrm{r}, \mathrm{p}}=\sqrt{e^{2}+i^{2}}\left[\frac{\boldsymbol{E}(k)}{\boldsymbol{K}(k)}\right]^{1 / 2} .
$$

In the above $\boldsymbol{K}(k)$ and $\boldsymbol{E}(k)$ are the complete elliptic integral of the first and second kind, respectively, with

$$
k=\sqrt{\frac{3 e^{2}}{4\left(e^{2}+i^{2}\right)}} .
$$

Considering the case that the gravitational energy of the planet $3 / a_{\mathrm{s}}$ is much larger than the kinetic energy of the planetesimal $v_{\mathrm{r}, \mathrm{p}}^{2} / 2$, we obtain $P_{\mathrm{s}} \propto a_{\mathrm{s}}^{-1}$. On the contrary, for larger $e$ and $a_{\mathrm{s}}, P_{\mathrm{s}}$ becomes independent of $a_{\mathrm{s}}$. Comparing $P_{\mathrm{s}, 2 \mathrm{~B}}$ with $P_{\mathrm{s}}$, we find $P_{\mathrm{s}}$ coincides well with $P_{\mathrm{s}, 2 \mathrm{~B}}$ for $\sqrt{e^{2}+i^{2}} \gtrsim 4.0$.

Property (2) can be interpreted from properties of retrograde orbits of planetesimals around the planet. In the case of $e=i=0$, we find that there are no retrograde orbits around the planet (i.e., orbits with $\dot{\varphi}<0$ at the time of collisions) with $r_{\text {min }} \gtrsim 0.06$, where $r_{\text {min }}$ denotes the minimum distance between a planetesimal and the planet, so that $a_{\mathrm{s}}$-dependency of $P_{\mathrm{s}}(e=i=0)$ changes near $a_{\mathrm{s}} \sim 0.06$; the detail is explained in Appendix B using the $b$-dependency of $r_{\text {min }}$. In the cases of small $e(\lesssim 1.0)$, we also find that each kink of $P_{\mathrm{s}}(e, i)$ corresponds to the maximum $r_{\min }$ of all retrograde orbits.

Next, we examine the collision rate of the satellite in planetesimal disks with nonuniform spatial distributions. With the decrease of $E_{\max }$, both $P_{\mathrm{s}}$ and $P_{\mathrm{p}}$ decrease because of

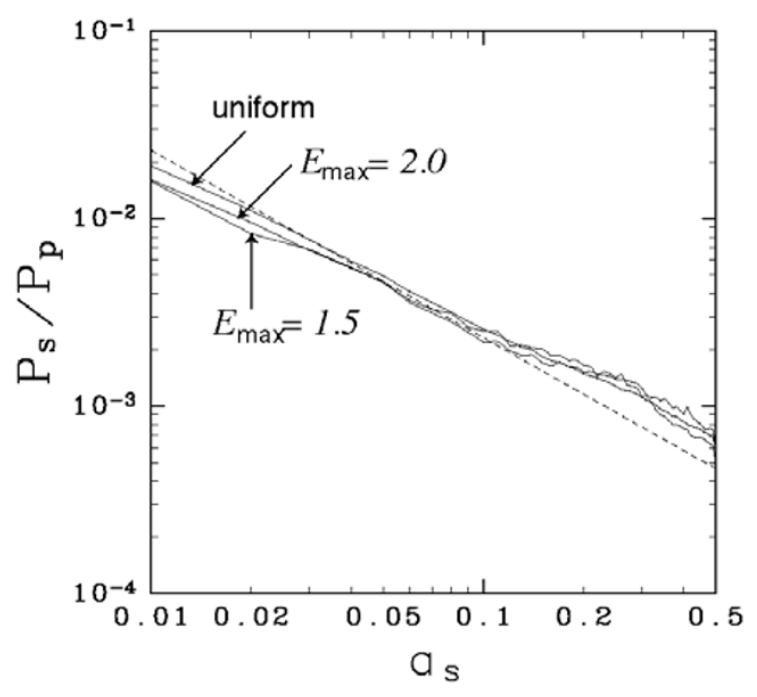

Fig. 4. Ratio of collision rate of the satellite $\left(\mu_{\mathrm{s}}=0.01\right)$ to that of the planet for two spatially nonuniform cases $\left(E_{\max }=1.5\right.$ and 2.0) and a uniform case with fixing all the cases as $(e, i)=(2,1)$. The dashed curve denotes analytic solution $P_{\mathrm{s}} / P_{\mathrm{p}}=r_{\mathrm{s}}^{2} /\left(r_{\mathrm{p}} a_{\mathrm{s}}\right)$ (see Eq. (23)).

broadening of the gap of planetesimals in the vicinity of the planet. For example, the values of $P_{\mathrm{s}}\left(e=2, i=1, a_{\mathrm{s}}\right)$ with $E_{\max }=1.5$ are reduced by a factor of about 20 from the values of $P_{\mathrm{s}}\left(e=2, i=1, a_{\mathrm{s}}\right)$ for the uniform case. Hence, Eq. (20) is no longer valid for the nonuniform disk. We are rather interested in the ratio $P_{\mathrm{s}} / P_{\mathrm{p}}$ than each absolute value, since the ratio determines the evolution of $\mu_{\mathrm{s}}$. Figure 4 shows $P_{\mathrm{s}} / P_{\mathrm{p}}$ as a function of $a_{\mathrm{s}}$ for $E_{\max }=1.5$ and 2.0 , and the uniform case with fixing all the cases as $(e, i)=(2.0,1.0)$; we find that $P_{\mathrm{s}} / P_{\mathrm{p}}$ is almost independent of $E_{\max }$. In fact, $P_{\mathrm{s}} / P_{\mathrm{p}}$ is well approximated by

$$
\left(\frac{P_{\mathrm{s}}}{P_{\mathrm{p}}}\right)_{2 \mathrm{~B}}=\left(\frac{r_{\mathrm{s}}}{r_{\mathrm{p}}}\right)^{2} \frac{\left(E_{\max }-9\right) / 2+3 / a_{\mathrm{s}}}{\left(E_{\max }-9\right) / 2+3 / r_{\mathrm{p}}},
$$

for $\sqrt{e^{2}+i^{2}} \gtrsim 2.0$. Since the kinetic energy of random motion of planetesimals, $\left(E_{\max }-9\right) / 2$, can be negligible compared with the planetary potential energy $3 / a_{\mathrm{s}}$ in the case of $\sqrt{e^{2}+i^{2}} \lesssim 4.0, P_{\mathrm{s}} / P_{\mathrm{p}}$ is almost independent of $E_{\max }$. In this case, Eq. (23) is simply described as $\left(P_{\mathrm{s}} / P_{\mathrm{p}}\right)_{2 \mathrm{~B}}=$ $r_{\mathrm{s}}^{2} /\left(r_{\mathrm{p}} a_{\mathrm{s}}\right)$, which is also shown as a dotted line in Fig. 4 .

\subsection{Specific angular momentum of planetesimals sup- plied to the satellite}

We here show the angular momentum supplied by planetesimals to the satellite. Neglecting planetary growth and tidal torque, we can say that $a_{\mathrm{s}}$ decreases with satellite growth when the specific angular momentum of the planetesimals $J_{\mathrm{s}}$ is smaller than unity. For the sake of comparison with $J_{\mathrm{s}}$, we also calculate mean specific angular momentum supplied to a fictitious circumplanetary disk with radius $a_{\mathrm{s}}$ after Herbert et al. (1986), which is given by

$$
=\frac{\int \frac{3}{2}|b| \sigma_{\mathrm{d}}(b) \frac{\ell_{z}}{\ell_{\mathrm{o}}}(e, i, b, \tau, \omega) p_{\mathrm{col}, \mathrm{d}}\left(e, i, b, \tau, \omega, a_{\mathrm{s}}\right) \frac{d \tau d \omega}{(2 \pi)^{2}} d b}{\int \frac{3}{2}|b| \sigma_{\mathrm{d}}(b) p_{\mathrm{col}, \mathrm{d}}\left(e, i, b, \tau, \omega, a_{\mathrm{s}}\right) \frac{d \tau d \omega}{(2 \pi)^{2}} d b},
$$




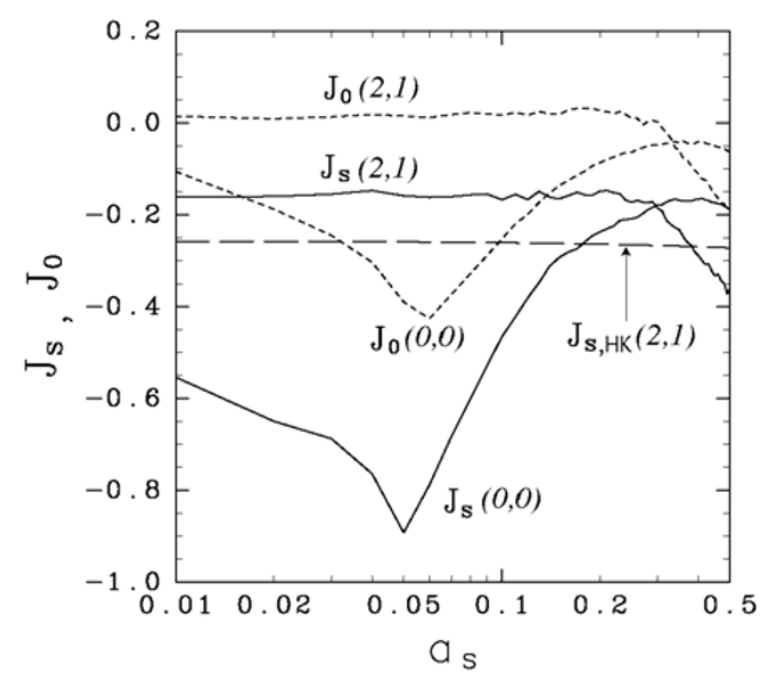

Fig. 5. Specific angular momentums supplied to the satellite $J_{\mathrm{S}}(e, i)$ and to a planetocentric disk $J_{0}(e, i)$ as functions of planetocentric distance $a_{\mathrm{s}}$ for the case of uniform spatial distribution of planetesimals. Analytic solution $J_{\mathrm{s}, \mathrm{HK}}$ given by Eq. (25) in the case of $(e, i)=(2,1)$ is also shown.

where $p_{\text {col,d }}$ is unity for crossing $(i=0)$ or penetrating $(i \neq$ $0)$ orbits and zero otherwise. It should be noted that putting $g_{3}=1$ (or $g_{2}=1$ ) in Eq. (12) (or in Eq. (16)) Eq. (11) reduces to Eq. (24). This means that the effect of the orbital motion of the satellite is not included in the value of $J_{0}$ whereas it is taken into account in the value of $J_{\mathrm{s}}$.

We first calculate $J_{\mathrm{s}}$ and $J_{0}$ for many pairs of $(e, i)$ for a planetesimal disk with uniform spatial distribution. Figure 5 shows $J_{\mathrm{s}}$ and $J_{0}$ as functions of $a_{\mathrm{s}}$ for the cases of $(e, i)=$ $(0,0)$ and $(e, i)=(2,1)$, respectively. For $(e, i)=(0,0)$, both $J_{0}$ and $J_{\mathrm{s}}$ are negative for all $a_{\mathrm{s}}$, which means that there exist more retrograde planetesimals than prograde ones. Behaviors of $J_{\mathrm{s}}$ and $J_{0}$ are similar and both have the minimum values near $a_{\mathrm{s}} \sim 0.06$. For larger $a_{\mathrm{s}}, J_{\mathrm{s}}$ and $J_{0}$ increase with $a_{\mathrm{s}}$. This is because number of collisional planetesimals with prograde orbits around the planet increases with $a_{\mathrm{s}}$, whereas number of retrograde orbits is kept constant. In Appendix B, we explain the detailed behavior of $J_{\mathrm{s}}(e=i=0)$ from $r_{\text {min }}(b)$ like that of $P_{\mathrm{s}}(e=i=0)$.

For $(e, i)=(2,1)$, both $J_{\mathrm{s}}$ and $J_{0}$ are constant when $a_{\mathrm{s}} \lesssim 0.30$, whereas they decrease with increasing $a_{\mathrm{s}}$ for larger $a_{\mathrm{s}}$. In such higher random-velocity cases incident of planetesimals is almost isotropic in the frame of reference of the planet, so that $J_{0}$ is almost 0 . However, $J_{\mathrm{s}}$ is negative even in these cases, since in the frame of reference of the satellite, incident of planetesimals is not isotropic due to orbital motion of the satellite; considering the relative azimuthal velocity of colliding planetesimals $\left(v_{\mathrm{r} \varphi}=\left|r \dot{\varphi}-r \omega_{\mathrm{S}}\right|\right)$, a retrograde planetesimal with $\dot{\varphi}(<0)$ has larger $v_{\mathrm{r} \varphi}$ than that of prograde one with same $|\dot{\varphi}|$, so that the enlargement factor $\left(g_{3}\right.$ in Eq. (13)) of collision probability for a retrograde planetesimal (it has negative $\ell_{z}$ ) is larger than that for the prograde counterpart (see Eq. (14)).

When incident of planetesimals is isotropic in the frame of reference of the planet, $J_{\mathrm{s}}$ is analytically obtained from Eqs. (9) and (12) of HK75:

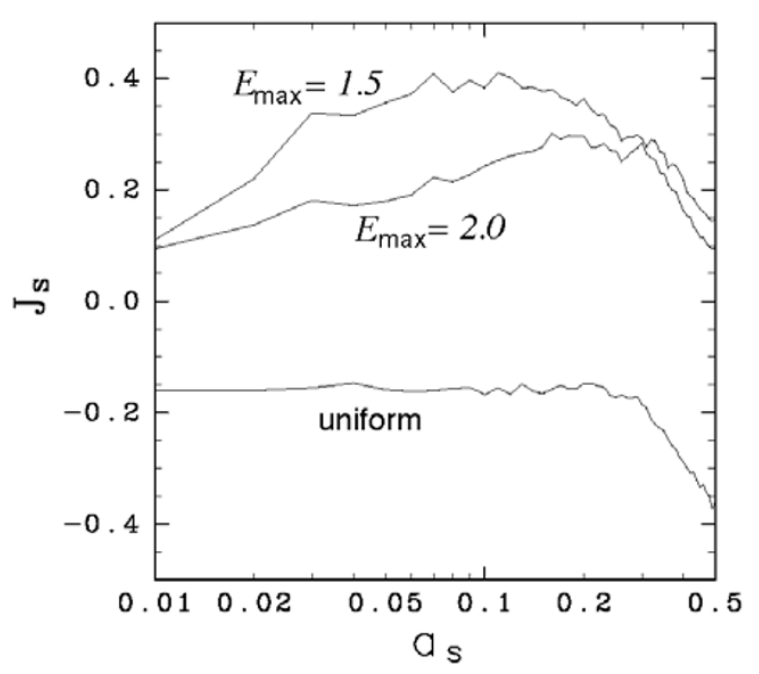

Fig. 6. Specific angular momentum supplied to the satellite $J_{\mathrm{s}}(e, i)$ for the case of nonuniform spatial distribution $\left(E_{\max }=1.5\right.$ and 2.0) of planetesimals with $(e, i)=(2,1)$. For comparison, the uniform case with the same $(e, i)$ is also shown.

$$
J_{\mathrm{s}, \mathrm{HK}}=-\frac{1}{3}\left(1+\frac{27}{5 a_{\mathrm{s}} v_{\mathrm{r}, \mathrm{p}}^{2}}\right)\left(1+\frac{7}{a_{\mathrm{s}} v_{\mathrm{r}, \mathrm{p}}^{2}}\right)^{-1}
$$

which is also shown as a long-dashed curve in Fig. 5. We find that $J_{\mathrm{s}, \mathrm{HK}}$ is almost independent of $a_{\mathrm{s}}$ and $v_{\mathrm{r}}$ and has a little deviation from the numerical results of $J_{\mathrm{s}}$. The deviation becomes smaller for larger $e$ if we focus on small $a_{\mathrm{s}}(\lesssim 0.1)$.

It should be noted that $J_{\mathrm{p}}(e, i)$ given in Eq. (8) and $J_{0}\left(e, i, a_{\mathrm{s}}\right)$ are the same quantity for the two-dimensional case. The previous studies showed that $J_{\mathrm{p}}$ is about -0.1 for $e=i=0$ (Ida and Nakazawa, 1990; Lissauer and Kary, 1991; Dones and Tremaine, 1993a; OI98), which well coincides with the value of $J_{0}$ with $a_{\mathrm{s}} \sim 0.01$. We also find that $J_{0}$ is almost zero for $e \gtrsim 2.0$, which is consistent with the previous studies of $J_{\mathrm{p}}$.

Next, we show the results of $J_{\mathrm{s}}$ for nonuniform disks. Figure 6 shows $J_{\mathrm{s}}$ as a function of $a_{\mathrm{s}}$ for two nonuniform cases with $E_{\max }=1.5$ and 2.0, and an uniform case. All of the cases, we fix $(e, i)=(2,1)$. In the nonuniform cases, $J_{\mathrm{s}}$ becomes positive for small $E_{\max }$, which means that there exist more prograde planetesimals than retrograde ones. Hence, in this case, accretion of planetesimals boosts up the angular momentum of the planet-satellite system. However, $J_{\mathrm{s}}$ never exceeds unity, so that $a_{\mathrm{s}}$ inevitably decreases with satellite growth. These results for small $E_{\max }$ are consistent with the case of planetary spin; the accretion of planetesimals with small $E_{\max }$ accounts for the rapid prograde spin of the planet, but the outcoming spin rate is much smaller than the value required for rotational instability (OI98).

\subsection{Evolution of planet-satellite systems}

We now investigate the accretional and dynamical evolution of planet-satellite systems. We assume that coalescence probability between the satellite and planetesimals is always unity. The effect of incomplete coalescence will be discussed in Subsection 5.2. 
The evolution of the mass ratio $\mu_{\mathrm{s}}$ can be expressed by

$$
\frac{1}{\mu_{\mathrm{s}}} \frac{d \mu_{\mathrm{s}}}{d t}=\frac{1}{\tau_{\text {grow }, \mathrm{p}}}\left(\frac{P_{\mathrm{s}}}{P_{\mathrm{p}} \mu_{\mathrm{s}}}-1\right),
$$

where $\tau_{\text {grow,p }}$ is the growth time of the planet given by

$$
\tau_{\text {grow }, \mathrm{p}}=\left(\frac{1}{M_{\mathrm{p}}} \frac{d M_{\mathrm{p}}}{d t}\right)^{-1}=\left(\frac{P_{\mathrm{p}} R_{\mathrm{H}}^{2} \Sigma_{\mathrm{d}}}{M_{\mathrm{p}}}\right)^{-1} .
$$

Here $\Sigma_{\mathrm{d}}$ is the surface density of planetesimals.

The evolution of the semimajor axis $a_{\mathrm{s}}$ can be expressed symbolically by

$$
\frac{1}{a_{\mathrm{s}}} \frac{d a_{\mathrm{s}}}{d t}=-\frac{1}{\tau_{\mathrm{acc}}}+\frac{1}{\tau_{\mathrm{tidal}}}-\frac{1}{\tau_{\mathrm{gas}}},
$$

where $\tau_{\text {acc }}, \tau_{\text {tidal }}$, and $\tau_{\text {gas }}$ are the time scales of accretion drag, tidal repulsion, and gas drag, respectively. To simplify the problem we here neglect the effect of gas drag (which will be discussed in Subsection 5.3). The effect of accretion drag is given by

$$
\frac{1}{\tau_{\text {acc }}}=\frac{1}{\tau_{\text {grow }, \mathrm{p}}}\left[\frac{2\left(1-J_{\mathrm{s}}\right) P_{\mathrm{s}}}{P_{\mathrm{p}} \mu_{\mathrm{s}}}+\frac{4}{3}\right],
$$

where first and second terms originate from accretion of planetesimals to the satellite and to the planet, respectively.

The satellite affects the repulsive force from the planet by tidal interaction when $a_{\mathrm{s}}$ is larger than the so-called synchronized radius $r_{\text {syn }}=\left(3 / \omega_{\mathrm{p}}^{2}\right)^{1 / 3}$, where $\omega_{\mathrm{p}}$ is the normalized spin angular velocity of the planet. The effect of tidal repulsion is given by (e.g., Mignard, 1979)

$$
\frac{1}{\tau_{\text {tidal }}}=3 \sqrt{3} \frac{k_{2}}{Q_{\mathrm{p}}} \frac{r_{\mathrm{p}}^{5} \mu_{\mathrm{s}}}{a_{\mathrm{s}}^{13 / 2}},
$$

where $k_{2}$ and $Q_{\mathrm{p}}$ are, respectively, the Love number of the second degree and the quality factor of the planet. Here $k_{2}$ for a homogeneous and incompressible planet can be estimated by (Love, 1934)

$$
k_{2}=\left(\frac{2}{5}+\frac{19}{15} \frac{\mu r_{\mathrm{p}}^{3}}{m_{\mathrm{p}}^{2 / 3}}\right)^{-1} \simeq k_{2 \mathrm{E}} m_{\mathrm{p}}^{2 / 3},
$$

where $\mu$ is the rigidity of the planet normalized by $\rho_{\mathrm{p}} R_{\mathrm{E}}^{2} \Omega_{\mathrm{k}}^{2}$, $R_{\mathrm{E}}$ and $k_{2 \mathrm{E}}$ are the radius and the Love number of the present Earth, respectively, and $m_{\mathrm{p}}=M_{\mathrm{p}} / M_{\mathrm{E}}$. The ratio of the two time scales $\tau_{\text {acc }} / \tau_{\text {tidal }}$ is given by

$$
\frac{\tau_{\mathrm{acc}}}{\tau_{\text {tidal }}}=3 \sqrt{3} \tilde{f} \frac{r_{\mathrm{p}}^{5} \mu_{\mathrm{s}} m_{\mathrm{p}}}{a_{\mathrm{s}}^{13 / 2}}\left[\frac{2\left(1-J_{\mathrm{s}}\right) P_{\mathrm{s}}}{P_{\mathrm{p}} \mu_{\mathrm{s}}}+\frac{4}{3}\right]^{-1}
$$

where $\tilde{f}$ denotes the tidal parameter defined by

$$
\tilde{f}=\frac{k_{2 \mathrm{E}}}{Q_{\mathrm{p}}} \tau_{\text {grow }, \mathrm{E}},
$$

with $\tau_{\text {grow, } \mathrm{E}}=\tau_{\text {grow, } \mathrm{p}} m_{\mathrm{p}}^{-1 / 3}$. Note that with increasing $\tilde{f}$, tidal repulsion becomes dominant in comparison with accretion drag. Taking the present values of parameters $k_{2 \mathrm{E}}=0.3$ and $Q_{\mathrm{p}}=10$, and the standard value $\tilde{\tau}_{\text {grow,E }}=$ (a)

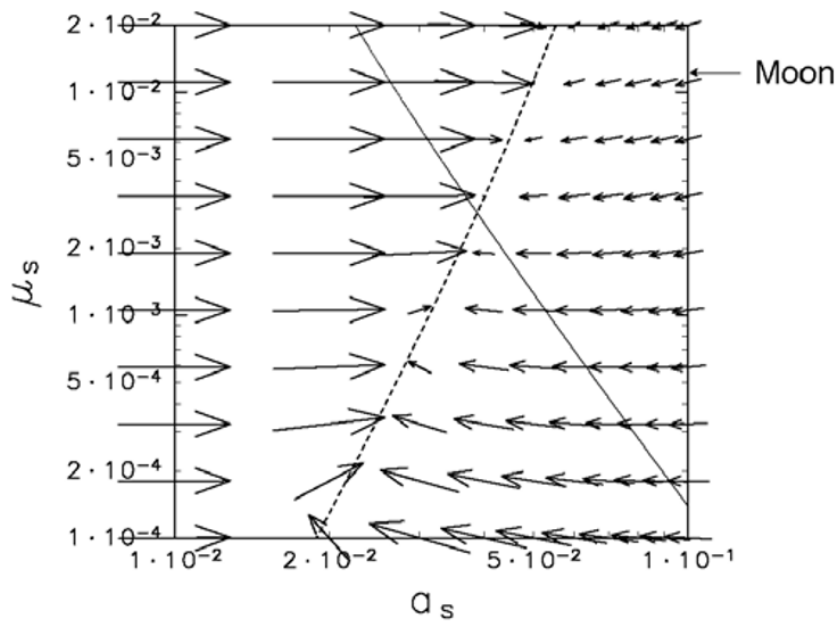

(b)

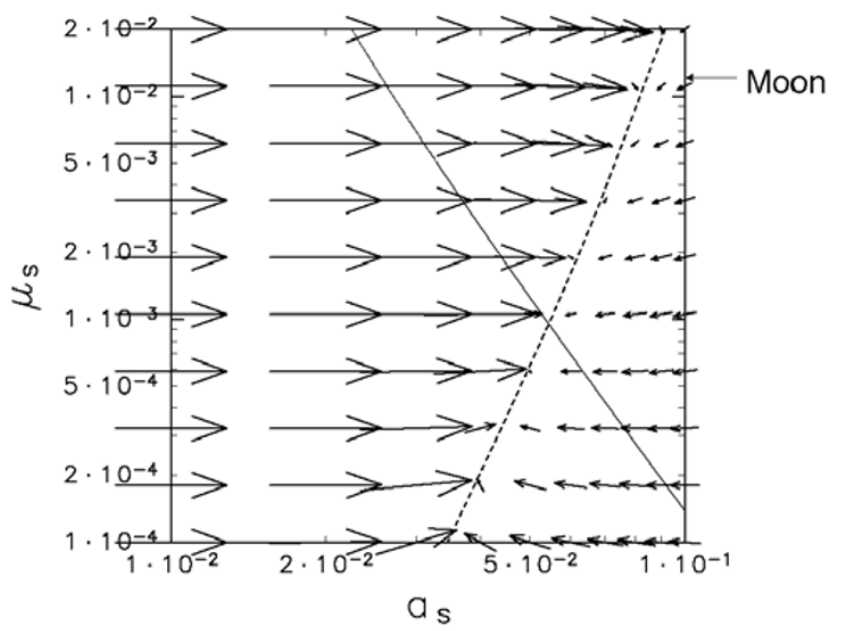

Fig. 7. Evolution of orbital radius $a_{\mathrm{s}}$ and mass ratio $\mu_{\mathrm{s}}$ of the satellite in the $a_{\mathrm{s}}-\mu_{\mathrm{s}}$ plane in the swarm of planetesimals with $(e, i)=(2,1)$. (a) Tidal parameter $f=0.21$, which corresponds to a spatially uniform disk. (b) $f=3.9$, which corresponds to a nonuniform disk with $E_{\max }=1.5$. Each vector component is proportional to inverse of corresponding evolutional time scale (but truncated by an upper limit). The solid curves show the stationary mass ratio where $d \mu_{\mathrm{s}} / d t=0$. The dashed curves show the balanced semimajor axis where $d a_{\mathrm{s}} / d t=0$. The satellite finally evolves into the equilibrium points where $d \mu_{\mathrm{s}} / d t=d a_{\mathrm{s}} / d t=0$.

$\tau_{\text {grow }, \mathrm{E}} \Omega_{\mathrm{K}}^{-1}(1 \mathrm{AU})=10^{6}$ years, we obtain $\tilde{f}=\tilde{f}_{0}=1.9 \times$ $10^{5}$. We re-normalize tidal parameter $\tilde{f}$ by $\tilde{f}_{0}$ as

$$
\begin{aligned}
f & =\frac{\tilde{f}}{\tilde{f}_{0}} \\
& =1.0\left(\frac{k_{2 \mathrm{E}} / Q_{\mathrm{p}}}{0.03}\right)\left(\frac{\Sigma_{\mathrm{d}}}{\Sigma_{0}}\right)^{-1}\left(\frac{P_{\mathrm{p}}}{6 \times 10^{-3}}\right)^{-1},
\end{aligned}
$$

where $\Sigma_{0}$ is the surface density of planetesimals for the minimum-mass solar nebula model at $1 \mathrm{AU}$ (Hayashi et al., 1985).

Here we assume that the planet spins so rapidly that exerting enough tidal repulsion. Figure 7 shows change rates of $a_{\mathrm{s}}$ and $\mu_{\mathrm{s}}$ in the plane of $a_{\mathrm{s}}$ vs. $\mu_{\mathrm{s}}$ with $m_{\mathrm{p}}=1.0,(e, i)=(2,1)$, 
and $\rho_{\mathrm{p}}=\rho_{\mathrm{s}}$. Further, we choose two types of spatial distributions of planetesimals, a uniform one (Fig. 7(a)) and a nonuniform one (Fig. 7(b)). The spatial distribution of planetesimals affects the normalized tidal parameter $f$ through $P_{\mathrm{p}}$; we find that Fig. 7(a) corresponds to the case of $f=0.21$ and Fig. 7(b) to $f=3.9$. The solid curves show the stationary mass ratio where $d \mu_{\mathrm{s}} / d t=0$. If $\mu_{\mathrm{s}}$ is smaller than the stationary mass ratio, $\mu_{\mathrm{s}}$ increases with growth, because smaller satellites have larger cross sections per mass. The dashed curves show the balanced semimajor axis where $d a_{\mathrm{s}} / d t=0$ (in other words, $\tau_{\text {acc }} / \tau_{\text {tidal }}=1$ ). The balanced semimajor axis for the nonuniform case is larger than that for the uniform case, because of the effects of larger $f$ (which comes from smaller $P_{\mathrm{p}}$ ) and larger $J_{\mathrm{s}}$ (see Fig. 6). Since the evolution time scale of $a_{\mathrm{s}}$ is generally much shorter than that of $\mu_{\mathrm{s}}, a_{\mathrm{s}}$ evolves toward the balanced semimajor axis quickly. Then, with keeping $a_{\mathrm{s}}$ to be the balanced semimajor axis, $\mu_{\mathrm{s}}$ evolves toward the stationary mass ratio. We denote the final locus the equilibrium point, where $d a_{\mathrm{s}} / d t=d \mu_{\mathrm{s}} / d t=0$, and its mass ratio $\mu_{\mathrm{s} \text {, eq. }}$.

Note that $\mu_{\mathrm{s} \text {, eq }}$ is the maximum mass ratio of the satellite if the satellite grows from a smaller embryo. We find that $\mu_{\mathrm{s} \text {,eq }}$ for the nonuniform case is smaller than that for the uniform case and both are smaller than $\mu_{\mathrm{M}}$. However, one should note that we show here the case with $(e, i)=(2,1)$ as a nominal example. In Section 4 we consider more realistic $(e, i)$ and examine whether $\mu_{\mathrm{s}, \text { eq }}$ exceeds $\mu_{\mathrm{M}}$.

In the case of a smaller planetary mass $\left(m_{\mathrm{p}}<1.0\right)$, the balanced radius becomes smaller (see Eq. (32)) and $\mu_{\mathrm{s} \text {,eq }}$ becomes larger in comparison with the case of $m_{\mathrm{p}}=1.0$. Also, the value of $f$ would not be a constant during the accretion. Thus, $\mu_{\mathrm{s} \text {,eq }}$ changes with growth, so that the final mass ratio $\mu_{\mathrm{s}}$ will deviate from $\mu_{\mathrm{s}, \text { eq }}$. Let us simply estimate how much the final $\mu_{\mathrm{s}}$ would deviate from $\mu_{\mathrm{s}, \mathrm{eq}}$, using Eq. (26). For the case that $\mu_{\mathrm{s}} \ll \mu_{\mathrm{s}, \mathrm{eq}}$, the time scale of the mass-ratio evolution (i.e., $\left.\mu_{\mathrm{s}}\left(d \mu_{\mathrm{s}} / d t\right)^{-1}\right)$ is much shorter

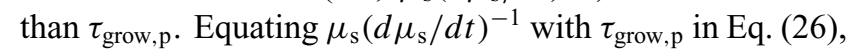
we find that within the time of $\tau_{\text {grow, }}, \mu_{\mathrm{s}}$ will be converge $\mu_{\mathrm{s} \text {,eq }}$ within a factor 2 in spite of the initial value of $\mu_{\mathrm{s}}$. Thus, we can roughly estimate the final mass ratios of long-lasting co-accretion cases from the mass ratio of the equilibrium point $\mu_{\mathrm{s} \text {,eq }}$ (see Subsection 4.1).

On the other hand, if $m_{\mathrm{p}}$ becomes unity and stop growing before $\mu_{\mathrm{s}}$ getting closer to $\mu_{\mathrm{s} \text {,eq }}$, the value of $\mu_{\mathrm{s}}$ at this point should be frozen as the final value. Thus, when discussing the co-accretion after late-stage formation of the satellite (such as the giant-impact hypothesis), we should directly deal with time evolution of $\mu_{\mathrm{s}}$ and $a_{\mathrm{s}}$ instead of using only $\mu_{\mathrm{s} \text {, eq }}$ (see Subsection 4.2).

\section{Two Types of Co-Accretion Scenarios for Lunar Origin}

In Subsection 3.3 we assumed that the planet spins so rapidly that tidal repulsion prevents the satellite from spiraling into the planet. We here demonstrate two types of promising situations which satisfy the rapid-spin condition and would bring forth the present Earth-Moon system. One is a long-lasting co-accretion case, in which an initial satellite embryo much smaller than the present Moon successively grew in a swarm of low-Jacobi-energy planetesimals in the solar nebula, and the other is an additional co-accretion case, in which a satellite as large as the present Moon was formed by a giant impact in the later stage, but not in the final stage, of Earth formation.

In both cases, the large angular momentum of the EarthMoon system $L_{\mathrm{EM}}$ as well as the large relative mass of the Moon $\mu_{\mathrm{M}}$ can be accounted for within possible ranges of initial masses and angular momentum. These ranges of initial values are important for us to constrain the conditions of possible Moon-forming events from the dynamical viewpoint.

It should be noted that if the Moon gained most of the mass during co-accretion, it might be hard to explain the bulk composition of the Moon, which is different from that of the Earth (e.g., Wood, 1986). It is a severe obstacle especially for a long-lasting co-accretion case. But it is not clear from the geochemical constraints how much the mass is allowed to accumulate onto the Moon during co-accretion. In order to overcome the geochemical constraints, one must first clarify a picture of accretional and dynamical evolution of co-accretion. So we here confine our discussion only to the dynamical constraints.

\subsection{Co-accretion from small embryos}

In this scenario, we consider that large spin angular momentum of the planet was supplied by a swarm of low-Jacobienergy planetesimals with nonuniform spatial distribution. OI98 showed that the final planetary spin rate by accretion of planetesimals with nonuniform spatial distribution of $E_{\max } \lesssim 1.5$ is large enough to explain $1 L_{\mathrm{EM}}$, where $E_{\max }$ is the maximum Jacobi integral of planetesimals (see Eq. (19)). Such lower values of the maximum Jacobi integral as well as lower random velocities is expected when the spatial and velocity distributions of the planetesimals are determined mainly by the balance between gravitational scattering by the planet and gas drag by the solar nebula.

We examine the case that a small satellite embryo evolves under the swarm of planetesimals with small $E_{\max }$. In Subsection 3.3, we found that the relative mass of the satellite evolves toward the equilibrium mass ratio $\mu_{\mathrm{s}, \mathrm{eq}}$. Inserting the relation $d \mu_{\mathrm{s}} / d t=d a_{\mathrm{s}} / d t=0$ into Eqs. (26) and (28) and using the relation $P_{\mathrm{s}} / P_{\mathrm{p}}=r_{\mathrm{s}}^{2} \alpha /\left(r_{\mathrm{p}} a_{\mathrm{s}}\right)$ (see Eq. (23)) (here we multiply the focusing factor of the satellite $\alpha=1+\left(v_{\text {esc }, \mathrm{s}} / v_{\mathrm{r}, \mathrm{s}}\right)^{2}$, which is not negligible for a lunarsized satellite), we can estimate the equilibrium mass ratio as

$$
\begin{aligned}
\mu_{\mathrm{s}, \mathrm{eq}} & =\left[\frac{2\left(1-J_{\mathrm{s}}\right)+4 / 3}{3 \sqrt{3} \tilde{f} m_{\mathrm{p}} r_{\mathrm{p}}^{5}}\right]^{6 / 19}\left(\frac{\rho_{\mathrm{s}}}{\rho_{\mathrm{p}}}\right)^{-26 / 19}\left(r_{\mathrm{p}} \alpha\right)^{39 / 19} \\
& =3.3 \times 10^{-3} f^{-6 / 19} m_{\mathrm{p}}^{-6 / 19}
\end{aligned}
$$

where we adopt appropriate values of parameters: $J_{s}=0.25$ (the averaged value for the case of $(e, i)=(2,1)$ and $E_{\max }=$ 1.5; see Fig. 6), $r_{\mathrm{p}}=0.004, \rho_{\mathrm{s}} / \rho_{\mathrm{p}}=0.6$ (the present values for the Earth-Moon system), and $\alpha=1.2$ (for the case of $\mu_{\mathrm{s} \text {, eq }}=\mu_{\mathrm{M}}$ ). Eq. (35) suggests that with decreasing $f$, the larger satellite is produced under the benefit of gravitational focusing by the planet, since the semimajor axis of the satellite is kept smaller. Choosing $m_{\mathrm{p}}=1.0$ and $f=3.9$, we find that $\mu_{\mathrm{s} \text {, eq }}$ is about one tenth of $\mu_{\mathrm{M}}$ (see Fig. 7b). Since $f$ depends on the uncertain values of the parameters $P_{\mathrm{p}}, \Sigma_{\mathrm{d}}$, and $Q_{\mathrm{p}}\left[f \propto\left(P_{\mathrm{p}} \Sigma_{\mathrm{d}} Q_{\mathrm{p}}\right)^{-1}\right]$, we here evaluate the proba- 
ble minimum value of $f$ at the later stage of Earth formation $\left(m_{\mathrm{p}} \sim 1.0\right)$ in order to check the possibility that $\mu_{\mathrm{s}, \text { eq }}$ reaches $\mu_{\mathrm{M}}$.

We first evaluate maximum value of the collision rate $P_{\mathrm{p}}$, which is a function of mean eccentricity $e$, inclination $i$, and maximum Jacobi integral $E_{\max }$ of planetesimals. In the later stage of planetary accretion, small fragments produced by mutual collisions of planetesimals are expected to make a main contribution to the planetary growth in the solar nebula (Wetherill and Stewart, 1993). The eccentricities and inclinations of these small fragments are estimated as $e \sim 3$ and $i \ll e$ under the balance between gravitational scattering by the proto-Earth and gas drag of the solar nebula (OI98). The condition that the final angular momentum of the EarthMoon system reaches $1 L_{\mathrm{EM}}$ restricts $E_{\max }$ must be smaller than 1.5. Under these values of $e, i$, and $E_{\max }$, we obtain the maximum value of $P_{\mathrm{p}}$ to be about 0.01 (OI98). It should be noted that such a large value of $P_{\mathrm{p}}$ can be acheived by the effect of small $i$ prevailing against that of small $E_{\max }$.

The maximum value of surface density $\Sigma_{\mathrm{d}}$ of solid material in the circumsolar disk can be somewhat larger than that of the minimum-mass model $\Sigma_{0}$ (Hayashi et al., 1985), whereas in the later stage of Earth formation it would decrease from the initial value. So we choose $\Sigma_{0}$ to be the maximum value of the surface density at the later stage of Earth formation.

We next estimate the maximum value of plausible $Q_{\mathrm{p}}$. The value for the present Earth is $Q_{\mathrm{p}} \sim 12$ (e.g., Lambeck, 1980; Burns, 1986); the main contribution is due to oceanic tides. Whereas $Q_{\mathrm{p}}$ would be 370 if solid tides only (Ray et al., 1996). The value of $Q_{\mathrm{p}}$ for a planet with the surface ocean is expected to increase with the depth of the ocean (Goldreich and Soter, 1966; Sagan and Dermott, 1982). In the course of planetary accretion we must consider the effect of the surface magma ocean, which would be formed when the mass of the proto-Earth exceeds 0.1 $M_{\mathrm{E}}$ (e.g., Kaula, 1979; Sasaki and Nakazawa, 1986). At the later stage of the Earth formation, the magma ocean would be deep and fully melted due to the strong green-house effect of the protoatmosphere (Abe and Matsui, 1985, 1986; Sasaki and Nakazawa, 1990) and the large values of estimated $P_{\mathrm{p}}$ (i.e., $\tilde{\tau}_{\text {grow,E }} \lesssim 10^{6}$ years). Hence, we expect that contribution of the magma ocean to tidal dissipation of the proto-Earth is rather small, so that we here adopt the maximum value of $Q_{\mathrm{p}}$ to be 400 .

It should be noted that the maximum values of the parameters given above were consistently realized if the Earth grew rapidly. Re-normalizing each parameter by the corresponding maximum value, we can rewrite Eq. (34) as

$$
f=0.015\left(\frac{Q_{\mathrm{p}}}{400}\right)^{-1}\left(\frac{\Sigma_{\mathrm{d}}}{\Sigma_{0}}\right)^{-1}\left(\frac{P_{\mathrm{p}}}{1 \times 10^{-2}}\right)^{-1} .
$$

Figure 8 shows the equilibrium points on the $\mu_{\mathrm{s}}$ vs. $a_{s}$ plane for $f=0.015$ and 1.0 with $m_{\mathrm{p}}=1.0, J_{\mathrm{s}}=0.25$, and $\rho_{\mathrm{s}} / \rho_{\mathrm{p}}=0.6$. Note that $\mu_{\mathrm{s}, \text { eq }}$ exceeds $\mu_{\mathrm{M}}$ in the case of $f=0.015$. In fact, $\mu_{\mathrm{s}, \mathrm{eq}} \sim \mu_{\mathrm{M}}$ when $f \sim 0.03$ (see Eq. (35)).

This means that, as long as from the dynamical viewpoint, the Moon could be grown up from a small embryo, if the following conditions were satisfied for the long-lasting coaccretion:

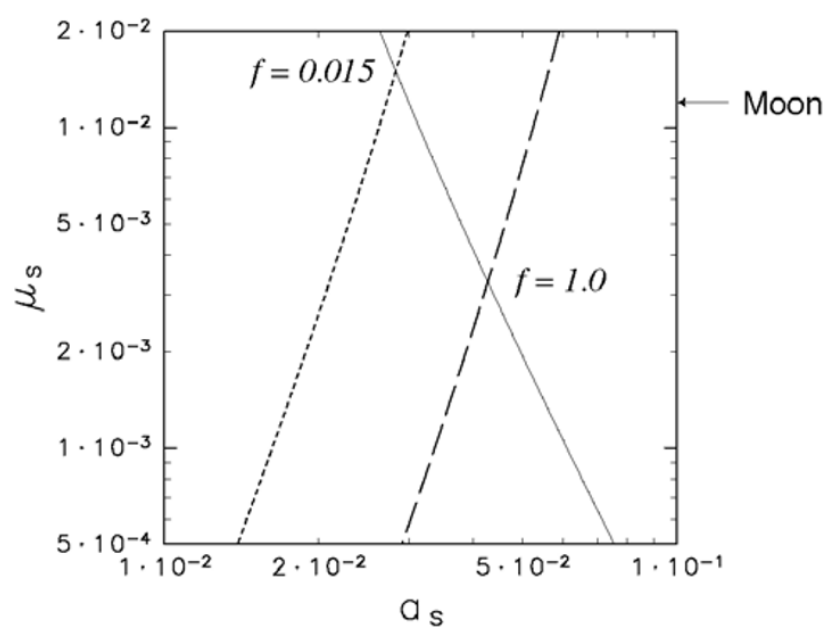

Fig. 8. Tidal-parameter $(f)$ dependency of the mass ratio at the equilibrium point $\mu_{\mathrm{s} \text {, eq }}$ in the $a_{\mathrm{s}}-\mu_{\mathrm{s}}$ plane. In the case of the minimum value of $f$ $\left(=0.015\right.$, see Eq. (37)), $\mu_{\mathrm{s}, \text { eq }}$ has the maximum value.

(1) The Earth and the Moon grew up in the solar nebula, so that the growth time of the Earth is short enough ( $\tilde{\tau}_{\text {grow,E }} \lesssim 10^{6}$ years) and that large angular momentum was supplied by planetesimals with small $E_{\max }$.

(2) Owing to fully-melted and deep magma ocean through accretion, $Q_{\mathrm{p}}$ of the proto-Earth was kept large $(\sim 400)$.

(3) The satellite avoided from spiraling into the planet owing to gas drag by the solar nebula (see Subsection 5.3).

These are rather strong dynamical constraints, so that we should examine whether these constraints are really satisfied in the course of Earth accretion. In the future work we will be able to clarify the possibility of realization of the long-lasting co-accretion from these dynamical constraints, besides from the frequentry-discussed geochemical constraints.

Until now, we focused on the final mass ratio of the satellite. Here, we estimate the minimum mass of an initial satellite embryo from which the Moon could grow up successively. As shown in Section 3, the embryo with small mass ratio grows around the balanced radius, which increases with the mass ratio. We can estimate the minimum mass by equating the balanced radius with the Roche radius $r_{\mathrm{R}} \sim 2.5 r_{\mathrm{p}} \sim$ 0.01 , within which planetary tides prevent the embryo from growing. Note that the synchronized radius is expected to be smaller than $r_{R}$, since planetary rotation is rapid enough due to accumlation of planetesimals with small $E_{\max }(\sim 1.5)$. The minimum mass $M_{\mathrm{s} \text {, min }}$ of the initial embryo is given approximately from Eqs. (23), (29), and (30) as

$$
\begin{aligned}
M_{\mathrm{s}, \min }= & 6.3 \times 10^{-3} M_{\mathrm{M}}\left(\frac{f}{0.015}\right)^{-3 / 4} \\
& \cdot\left(\frac{m_{\mathrm{p}}}{0.1}\right)^{1 / 4}\left(\frac{r_{\mathrm{R}}}{2.5 r_{\mathrm{p}}}\right)^{33 / 8} .
\end{aligned}
$$

Note that dependency of $M_{\mathrm{s} \text {, min }}$ on $m_{\mathrm{p}}$ is rather small, so that we can fairly constrain the minimum mass of the lunar embryo. Though HK75 obtained a similar expression of $M_{\mathrm{s}, \mathrm{min}}$, their estimated mass of the initial embryo is much 
smaller than ours. This is because their adopted random velocity of planetesimals was larger (i.e., smaller $P_{\mathrm{p}}$ ) and $Q_{\mathrm{p}}$ was smaller than ours.

\subsection{Co-accretion after the Moon-forming giant impact}

Recently, the Moon-forming giant impact is considered to have occurred during Earth accretion (Cameron and Canup, 1998a, b; Canup et al., 2001). In this scenario, the large spin angular momentum as well as a lunar-sized satellite were produced by the giant impact itself. Then, the mass ratio and the angular momentum of the Earth-Moon system would be changed through co-accretion thereafter. After the dissipation of the solar nebular, random velocities of planetesimals were determined by the balance between gravitational scattering by the planet and dissipation due to mutual inelastic collisions. The balanced values of the random velocities become larger than those in gaseous environment, so that net angular momentum brought by planetesimals was almost zero and the planet would spin down in the post giant-impact stage.

In order to confine dynamical parameters (e.g., mass ratio, angular momentum) immediately after the giant impact, we go back evolution of the Earth-Moon system from the final stage of Earth formation to the past. The changes of $\mu_{\mathrm{s}}$ and $a_{\mathrm{s}}$ can be expressed by Eqs. (26) and (28), respectively, and the change of $\omega_{\mathrm{p}}$ by

$$
\frac{1}{\omega_{\mathrm{p}}} \frac{d \omega_{\mathrm{p}}}{d t}=-\frac{5}{3} \frac{1}{\tau_{\text {grow }, \mathrm{p}}}-\frac{1}{2} \frac{\mu_{\mathrm{s}} \sqrt{3 a_{\mathrm{s}}}}{I r_{\mathrm{p}}^{2} \omega_{\mathrm{p}}} \frac{1}{\tau_{\text {tidal }}},
$$

where $I$ is the normalized moment of inertia of the planet (we here adopt $I=0.33$, the value for the present Earth). In Eq. (38), the first term of the right-hand side denotes the effect of planetesimal accretion and the second term the effect of tidal interaction with the satellite. We also calculate the total angular momentum of the planet-satellite system, $L_{\mathrm{ps}}=$ $I M_{\mathrm{p}} R_{\mathrm{p}}^{2} \omega_{\mathrm{p}} \Omega_{\mathrm{K}}+M_{\mathrm{s}} \sqrt{G M_{\mathrm{p}} A_{\mathrm{s}}}$. We choose the starting values of the mass ratio to be $\mu_{\mathrm{M}}$, the total angular momentum to be $L_{\mathrm{EM}}$, and $a_{\mathrm{s}}$ to be the balanced radius $a_{\mathrm{s}, \text { ba }}$ for $\mu_{\mathrm{s}}=\mu_{\mathrm{M}}$ (see Subsection 3.3).

We halt each backward integration if one of the following conditions is satisfied:

$$
\begin{aligned}
& \text { (1) } a_{\mathrm{s}}>a_{\mathrm{s}, \mathrm{ba}}, \\
& \text { (2) } a_{\mathrm{s}}<r_{\mathrm{R}}, \\
& \text { (3) } \omega_{\mathrm{p}}>5600 .
\end{aligned}
$$

Condition (3) corresponds to the marginal value of the secular bar instability of a rapidly rotating planet (Durisen and Gingold, 1986). During the integration we keep the semimajor axis of the satellite to be the balanced radius at each time; otherwise the integration would halt more rapidly.

We determine the parameters for the integration, i.e., the mean random velocity, the surface density of planetesimals, and the specific angular momentums supplied to the planet and the satellite, as follows.

The mean random velocity $v_{\mathrm{r}}$ of planetesimals in gasfree environment is given by the balance between gravitational scattering by the planet and dissipation due to mutual inelastic collisions. The time scale of increasing of $v_{\mathrm{r}}$ by planet's gravitational scattering (i.e., the viscous stirring time) is given by (Ida, 1990; Ida and Makino, 1993)

$$
T_{\mathrm{vs}}=\frac{c_{\mathrm{e} 1}}{n_{\mathrm{p}} r_{\mathrm{p}}^{2}}\left(\frac{v_{\mathrm{r}}}{v_{\mathrm{esc}, \mathrm{p}}}\right)^{4},
$$

where $c_{\mathrm{e} 1}$ is a numerical factor $(\sim 0.10), n_{\mathrm{p}}$ is the effective surface number density of protoplanets (one of which is the proto-Earth), and $v_{\mathrm{esc}, \mathrm{p}}(\sim 35)$ is the escape velocity of the planet. On the other hand, the time scale of mutual collisions of planetesimals is given by (Greenzweig and Lissauer, 1990; Ohtsuki, 1993)

$$
T_{\mathrm{col}}=\frac{c_{\mathrm{e} 2}}{n_{\mathrm{d}} r^{2}},
$$

where $c_{\mathrm{e} 2}$ is a numerical factor $(\sim 0.54), n_{\mathrm{d}}$ is the surface number density of planetesimals, and $r$ is the sum of planetesimal radii. Here we assumed that $e=2 i$ and that masses of all the planetesimals are equal. Equating $T_{\mathrm{vs}}$ with $T_{\text {col }}$, we obtain

$$
v_{r}=0.4\left(\frac{n_{\mathrm{p}} M_{\mathrm{p}} / \Sigma_{\mathrm{d}}}{2.0}\right)^{1 / 4}\left(\frac{m / M_{\mathrm{p}}}{10^{-6}}\right)^{1 / 12} v_{\mathrm{esc}, \mathrm{p}},
$$

where $m$ is the mass of planetesimals. According to the above equation, we here choose the value of eccentricity and inclination of planetesimals as $(e, i)=(15,7.5)$.

We assume that the surface density of planetesimals is kept constant with the value of the minimum-mass model during the growth for simplicity. Then in gas-free environment, the growth time of the Earth given by Eq. (27) is about $6.0 \times 10^{7}$ years, which is two orders of magnitude longer than that for accretion in the gaseous environment adopted in Subsection 4.1.

Because of the large random velocities of planetesimals, we adopt that specific angular momentums supplied to the planet and to the satellite are, respectively, $J_{\mathrm{p}}=0$ and $J_{\mathrm{s}}=$ -0.2 (see Subsection 3.2), which are fairly smaller than those in the gaseous environment $\left(J_{\mathrm{p}} \sim 0.2, J_{\mathrm{s}} \sim 0.1\right)$.

Using these parameters, we perform the integration. Figure 9 shows the results; changes of $\ell_{\mathrm{ps}}\left(=L_{\mathrm{ps}} / L_{\mathrm{EM}}\right), \omega_{\mathrm{p}}$, $a_{\mathrm{s}}$, and $\mu_{\mathrm{s}}$ as functions of $m_{\mathrm{p}}$ for two different values of $f=60$ (long-dashed curves) and 1.5 (solid curves), which correspond to the cases of $Q_{\mathrm{p}}=10$ and 400, respectively. In both cases the backward integrations were halted by satisfying Condition (3) (see Fig. 9(b)) when $m_{\mathrm{p}} \sim 0.55$, because a smaller planet cannot have angular momentum as large as that of the Earth-Moon system. Hence, the giant impact should occur after the mass of the proto-Earth exceeds about half of the present mass.

Figure 9(a) shows that $\ell_{\mathrm{ps}}$ decreases with increasing $m_{\mathrm{p}}$. This is because of collisional-planetesimals anisotropy due to the orbital motion of the satellite (see Subsection 3.2). We find that the angular momentum supplied by the giant impact should be a factor $0.5\left|J_{\mathrm{s}}\right|\left(1-m_{\mathrm{p}}\right)$ (for $m_{\mathrm{p}} \sim 0.5$, about $5 \%$ ) larger than $1 L_{\mathrm{EM}}$. The giant-impact simulations by Cameron and Canup (1998a) in the case of $m_{\mathrm{p}}=0.6$ (where we use $m_{\mathrm{p}}$ as the total mass of the impactor and the target) show that mass of the disk generated by an impact with the total angular momentum $1.2 L_{\mathrm{EM}}$ is twice as large as that with $1.0 L_{\mathrm{EM}}$. Thus, even $5 \%$ increase of total angular momentum as shown in Fig. 9(a) would be effective for forming a larger 
(a)

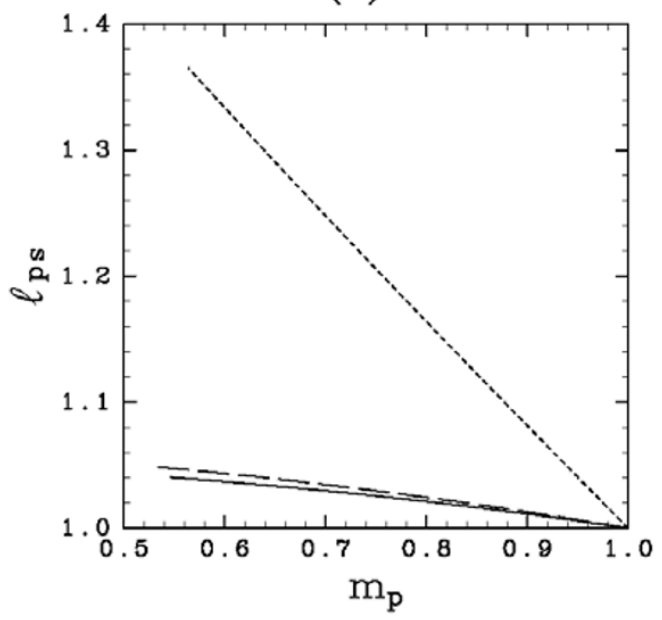

(c)

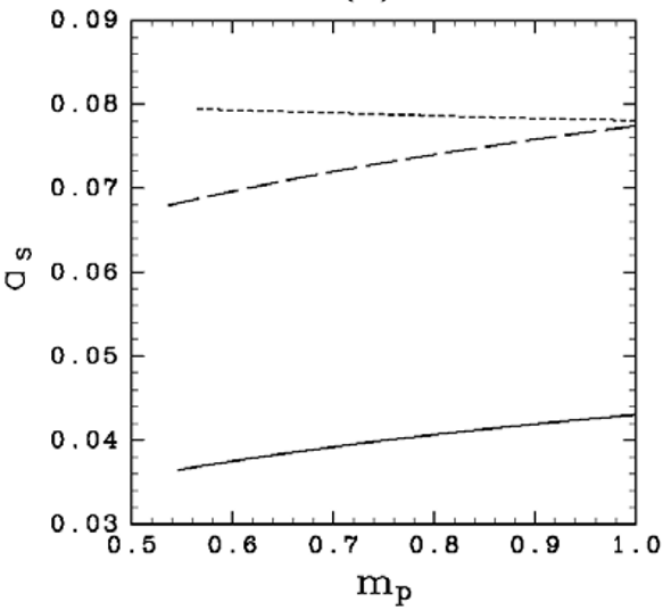

(b)

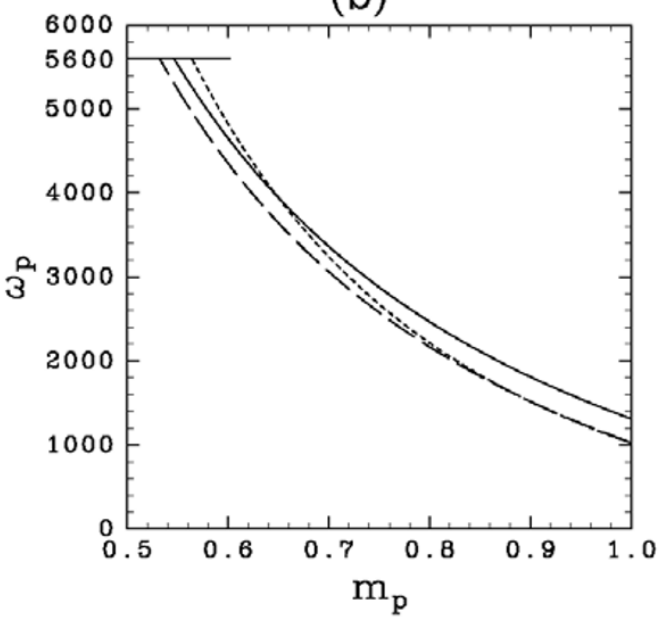

(d)

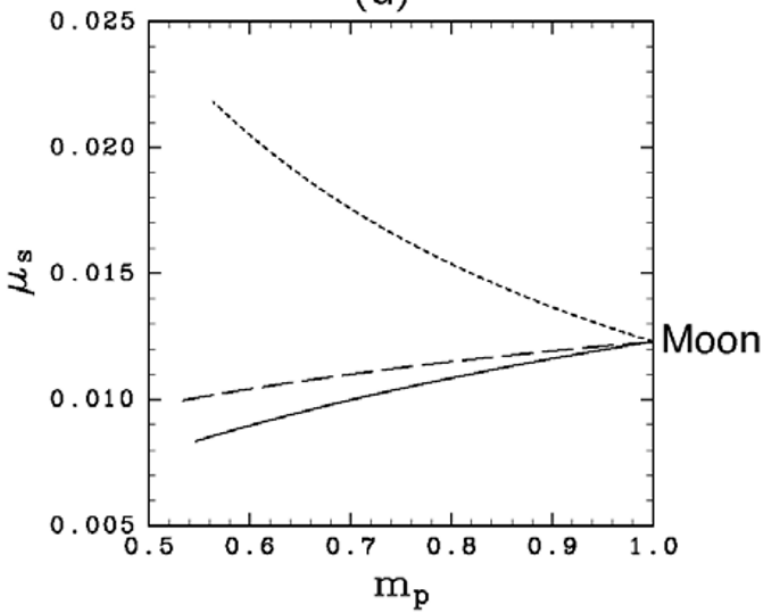

Fig. 9. Evolutions of total angular momentum $\ell_{\mathrm{ps}}(\mathrm{a})$, planet's spin angular velocity $\omega_{\mathrm{p}}(\mathrm{b})$, satellite's planetocentric distance $a_{\mathrm{s}}$ (c), and relative mass $\mu_{\mathrm{s}}(\mathrm{d})$ as functions of planet mass for two perfect accretion cases with $f=1.5$ (solid curves) and 60 (long-dashed curves), and one non accretion case $\left(d M_{\mathrm{s}} / d t=0\right)$ with $f=60$ (short-dashed curves). The integrations start from $m_{\mathrm{p}}=1.0$ to the past. The starting values for $\mu_{\mathrm{s}}$ and $\ell_{\mathrm{ps}}$ are the present values of the Earth-Moon system $\left(\mu_{\mathrm{s}}=\mu_{\mathrm{M}}, \ell_{\mathrm{ps}}=1.0\right)$, but $a_{\mathrm{s}}$ is determined from a given value of $f\left(a_{\mathrm{s}}=a_{\mathrm{s}, \mathrm{ba}}\right)$ and $\omega_{\mathrm{p}}$ is determined from all these values. In all the cases, the backward integrations are halted where rotational instability of the planet will occur $\left(\omega_{\mathrm{p}}>5600\right)$.

protoMoon if co-accretion after the giant impact is taken into account.

Figure 9(d) shows that $\mu_{\mathrm{s}}$ increases with $m_{\mathrm{p}}$. This is because the Moon has larger collision cross section per mass than that of the Earth. In other words, $\mu_{\mathrm{s} \text {, eq }}$ for the case of larger $v_{\mathrm{r}}$ (as adopted in Fig. 9) exceeds $\mu_{\mathrm{M}}$ even for the case of larger $f$. The value of $\mu_{\mathrm{s}}$ increases more rapidly with decreasing $f$ because a satellite with smaller balanced radius (see Fig. 9(c)) receives a larger benefit of planetary focusing. In any cases, however, mass-ratio changes are rather small compared with the situation in Subsection 4.1 because of smaller mass change of the Earth itself.

Until now, we assumed that the coalescence probability between the satellite and planetesimals to be unity. But in high-speed collisions, the total mass of escaping ejecta from the satellite might become comparable to the mass of a impactor (for a full discussion of ejecta, see Subsection 5.2). Here we also calculate the post-giant-impact co-accretion in an extreme case that $d M_{\mathrm{s}} / d t=0$. We assume that all of escaping ejecta from the satellite also escape from the planetary Hill sphere and that their mean specific geocentric angular momentum is same as that of the satellite. We perform integration of the co-accretion evolution backward from $m_{\mathrm{p}}=1.0$ for the case of $f=60$. The results are also shown in Fig. 9 (short-dashed curves). Figure 9(a) shows that $\ell_{\mathrm{ps}}$ decreases with increasing $m_{\mathrm{p}}$ much faster than the perfect accretion cases, since escaping ejecta take away large amount of angular momentum from the planet-satellite system. With a increase in total mass of escaping ejecta from the satellite, larger $\ell_{\mathrm{ps}}$ is allowed immediately after the giant impact.

\section{Discussion}

\subsection{Comparison with results of giant-impact simula-} tions

In Subsection 4.2, starting from the present values of the 


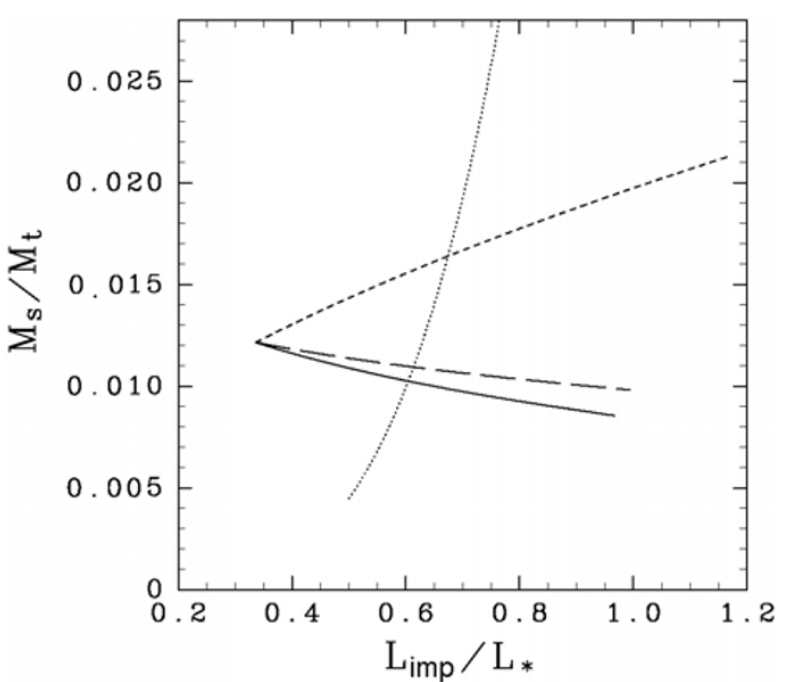

Fig. 10. Mass ratio of a impact-generated satellite relative to total colliding mass as a function of impact angular momentum normalized by $L_{*}$ (dotted curve) with fixing an impactor-to-target mass ratio to be 3:7 and an impact velocity to be the mutual escape velocity. Three evolutional curves, which correspond to the results shown in Fig. 9 (solid, short-dashed, and long-dashed curves), are also shown. The converging point of three curves corresponds to the present state of the Earth-Moon system.

mass ratio and the total angular momentum, we went back the evolution of the Earth-Moon system to the past. Thus, if all the dynamical quantities of the outcome of a giantimpact simulation locate on the evolution curves of the corresponding quantities obtained in our calculation, the generated system will finally reach to the present Earth-Moon system through co-accretion. Here we compare our results with the results of the giant-impact simulations.

Canup et al. (2001) compiled the results of the impact simulations, all employing an impactor-to-target mass ratio of 3:7 and equalizing the impact velocity with the mutual escape velocity of the target and the impactor. They showed that mass of the impact-generated satellite $M_{\mathrm{S}}$ is given by

$$
\begin{aligned}
& \frac{M_{\mathrm{s}}}{M_{\mathrm{t}}}=\left[0.15\left(\frac{L_{\mathrm{imp}}}{L_{*}}\right)^{3.83}-0.064\left(\frac{L_{\text {imp }}}{L_{*}}\right)^{3.40}\right] \\
& \left(0.5<\frac{L_{\text {imp }}}{L_{*}}<1.0\right) \text {, }
\end{aligned}
$$

where $L_{\mathrm{imp}}$ and $M_{\mathrm{t}}$ are the impact angular momentum and the total mass of the impactor and the target, and $L_{*}=$ $2.91\left(M_{\mathrm{t}} / M_{\mathrm{E}}\right)^{5 / 3} L_{\mathrm{EM}}$ denotes the planetary angular momentum spinning at the maximum rate for rotational instability.

Figure 10 shows the relation between $M_{\mathrm{s}} / M_{\mathrm{t}}$ and $L_{\mathrm{imp}} / L_{*}$ given by Eq. (42) (dotted curve). It is clear that $M_{\mathrm{s}} / M_{\mathrm{t}}$ drastically increases with $L_{\text {imp }} / L_{*}$. In the same figure, we also re-plot three evolution curves shown in Fig. 9. Note that the converging point of the three curves corresponds to the present state of the Earth-Moon system. An important feature of this figure is that mass of a satellite formed by a impact with the present total mass and the angular momentum of the Earth-Moon system is much smaller than that of the Moon. This problem has been discussed by multiple previous works (Canup and Esposito, 1996; Ida et al., 1997; Cameron, 1997, Cameron and Canup, 1998a, b; Canup et al., 2001). If smaller total mass is considered, larger satellite mass is achieved owing to increasing of $L_{\text {imp }} / L_{*}$. We find each evolution curve has a point of intersection with the curve represented by Eq. (42). It should be noted that if a giant impact produces a planet-satellite system corresponding to each point of intersection, the generated system evolves into the Earth-Moon system through co-accretion with the corresponding conditions. From Fig. 9(d), we can find that the value of $m_{\mathrm{p}}$ of at the three points of intersection are almost equal to 0.7 . This suggests that the Moon-forming impact was occurred when $M_{\mathrm{t}} \simeq 0.7 M_{\mathrm{E}}$, regardless of the conditions of subsequent co-accretion stage.

However, Eq. (42) is confined to the case that the impactorto-target mass ratio to be $3: 7$ and the impact velocity to be the mutual escape velocity. Giant-impact simulations in wider parameters region are desirable.

\subsection{Coalescence probability between the satellite and planetesimals}

In most of the calculations, we assumed that coalescence probability between the satellite and planetesimals to be unity. It might fairly decrease in high-speed collisions. Here, we estimate the coalescence probability $P_{\text {co }}$, i.e.,

$$
P_{\mathrm{co}}=1-\frac{m_{\mathrm{esc}}}{m}\left(1-q_{\mathrm{re}}\right),
$$

where $m$ and $m_{\text {esc }}$ denote, respectively, the colliding planetesimal mass and the total mass of ejecta escaping from the satellite, and $q_{\text {re }}$ is the mass fraction of re-accumulating ejecta which once escaped from the gravitational sphere of the satellite to the circumplanetary orbit.

Using the scaling laws of impact cratering in the gravity regime given by Housen et al. (1983), we estimate the escaping mass $m_{\text {esc }}$ as a power-law function of impact velocity of the planetesimal $v_{\text {imp }}=\left(v_{\mathrm{r}}^{2}+9 / a_{\mathrm{s}}+v_{\mathrm{esc}, \mathrm{s}}^{2}\right)^{1 / 2}$. Adopting the coefficients and the power-law indices determined from laboratory experiments of sand targets (Holsapple, 1993; Housen et al., 1983), we obtain

$$
\frac{m_{\mathrm{esc}}}{m}=0.12\left(\frac{v_{\mathrm{imp}}}{v_{\mathrm{esc}, \mathrm{s}}}\right)^{1.23} .
$$

Setting $a_{\mathrm{s}}$ to be the balanced radius, we obtain $v_{\text {imp }} \sim$ $2.3 v_{\mathrm{esc}, \mathrm{s}}$ for $\mu_{\mathrm{s}}=\mu_{\mathrm{M}}$ and $v_{\mathrm{imp}} \sim 12.5 v_{\mathrm{esc}, \mathrm{s}}$ for $\mu_{\mathrm{s}}=$ $M_{\mathrm{s}, \min } /\left(0.1 M_{\mathrm{E}}\right)$ in the gaseous environment discussed in Subsection 4.1. In the gas-free environment discussed in Subsection 4.2, we have $v_{\text {imp }} \sim 2.6-3.2 v_{\text {esc,s. }}$. Putting these values in Eq. (44), we have $m_{\text {esc }} / m \sim 0.33-2.7$ and 0.39 0.50 , respectively. A small initial embryo as evaluated in Eq. (37) ejects mass more than impactor mass, which would prevent the satellite growth $\left(P_{\mathrm{co}}<0\right)$, if $q_{\mathrm{re}}$ is small enough. Hence, the minimum mass of the initial embryo might be larger than that in the perfect accretion case. On the other hand, even for $q_{\mathrm{re}}=0$, the satellite with the mass ratio as large as that of the Moon can safely grow $\left(P_{\mathrm{co}}>0\right)$ in both situations in Subsection 4.1 and Subsection 4.2, though the growth rate would be reduced. For the situation in Subsection 4.1, we estimate the $\mu_{\mathrm{s} \text {, eq }}$ again adopting imperfect accretion given by Eqs. (43) and (44) with $q_{\mathrm{re}}=0$, and find that the condition $\mu_{\mathrm{s} \text {,eq }}>\mu_{\mathrm{M}}$ is still satisfied for $f \sim 0.015$. Note that, however, the coefficient as well as the power-law in- 
dex of the right-hand side of Eq. (44) have some uncertainty, which would affect the conclusion.

Even in the case that the escape mass is comparable to the impactor mass, the satellite can grow if most of the ejecta remain in the Hill sphere of the planet and finally re-accumulate onto the satellite. Though most of the ejecta escaping from the present Moon $\left(a_{\mathrm{s}}=0.25\right)$ also escape from the Hill sphere of the Earth (Gladman et al., 1995), re-accumulation fraction might be higher in the co-accretion stage, when the Moon was much closer to the Earth.

Ejecta thrown in orbits around the planet are gravitationally scattered by the satellite and the velocity dispersion of the ejecta increases to almost as large as the escape velocity of the satellite. Then, if the orbital velocity of the satellite around the planet is larger than the escape velocity of the satellite, we can safely assume that the ejecta finally reaccumulates to the satellite without escaping from the Hill sphere of the planet or fall onto the planet. This condition can be written as $a_{\mathrm{s}}<0.04\left(\mu_{\mathrm{s}} / \mu_{\mathrm{M}}\right)^{-2 / 3}$. For the situations in Subsection 4.1, the re-accumulation condition is satisfied for a satellite with $\mu_{\mathrm{s}} \lesssim \mu_{\mathrm{s} \text {,eq }} \simeq \mu_{\mathrm{M}}$, so that the value of $P_{\text {co }}$ would be close to unity even if $m_{\text {esc }} / m$ is not so small. On the other hand, the condition of re-accumulation is not satisfied for the situation in Subsection 4.2. Hence, the value of $P_{\text {co }}$ would be very small if $m_{\text {esc }} / m$ is almost unity.

In order to obtain more reliable values of the re-accumulation fraction $q_{\text {re }}$, we also performed many orbital calculations of escaping ejecta, details of which will be presented in the next paper (Morishima and Watanabe, in preparation). We found that $q_{\mathrm{re}}$ for a satellite with $a_{\mathrm{s}}>0.04\left(\mu_{\mathrm{s}} / \mu_{\mathrm{M}}\right)^{-2 / 3}$ is small $(\lesssim 0.2)$ and that most of the escaping ejecta from the satellite escape also from the Hill sphere of the planet (its fraction is $\gtrsim 0.7$ ). We also found that the mean specific angular momentum of escaping ejecta from the planetary Hill sphere is about 1.2-1.3 times larger than that of the satellite. Since decreasing rate of angular momentum by the escaping ejecta is proportional to a product of the mass fraction of escaping ejecta and their mean specific angular momentum, the assumptions adopted in Subsection 4.2 is fairly good.

\subsection{Gas drag on the satellite}

Since we have adopted gaseous environment in the situation discussed in Subsection 4.1, we should estimate the effects of gas drag on the satellite. After Adachi et al. (1976), the decay time of the semi-major axis of a satellite by gas drag is given by

$$
\tau_{\text {gas }}=\left|a_{\mathrm{s}}\left(\frac{d a_{\mathrm{s}}}{d t}\right)_{\mathrm{gas}}^{-1}\right|=\frac{4}{3} \frac{\eta^{2} r_{\mathrm{s}}}{C_{\mathrm{D}} v_{\mathrm{s}}} \frac{\rho_{\mathrm{s}}}{\rho_{\mathrm{g}}},
$$

where $C_{\mathrm{D}}$ is the gas drag coefficient $(\sim 0.5)$ and $\rho_{\mathrm{g}}$ is the gas density around the satellite. Further, $\eta$ is the fractional deviation of the gas velocity from the local Keplerian velocity around the planet $\left(\eta=1-v_{\mathrm{g}} / v_{\mathrm{s}}\right.$, where $v_{\mathrm{g}}$ denotes the rotational gas velocity).

We estimate $\tau_{\text {gas }}$ for the two types of gaseous environment around the satellite: (1) a hydrostatic atmosphere $(\eta=1)$ and (2) a Keplerian subdisk $(\eta \ll 1)$; it is controversial which type is more plausible.

First we consider the hydrostatic atmosphere. When the planet mass becomes greater than that of the present Moon, gas around the planet is getting denser than ambient solar nebula and is well regarded that the planet has an atmosphere. The outer boundary of the atmosphere is the Bondi radius $r_{\mathrm{B}}$ defined by

$$
r_{\mathrm{B}}=3 / c^{2}=0.25 m_{\mathrm{p}}^{2 / 3},
$$

where $c$ is the sound velocity of the gas, and the temperature is assumed to be $280 \mathrm{~K}$. The structure of the hydrostatic atmosphere was obtained by Sasaki and Nakazawa (1990). Assuming radiative equilibrium and a constant opacity, the density structure is given analytically by

$$
\rho_{\mathrm{g}, \mathrm{st}}\left(a_{\mathrm{s}}\right)=\rho_{\mathrm{q}}\left[\left(\frac{r_{\mathrm{p}}}{a_{\mathrm{s}}}\right)^{3}-\left(\frac{r_{\mathrm{p}}}{r_{\mathrm{B}}}\right)^{3}\right]+e \rho_{\mathrm{g} 0},
$$

where $\rho_{\mathrm{g} 0}$ is the gas density of the minimum-mass solar nebula model at $1 \mathrm{AU}$ (Hayashi et al., 1985). Further $\rho_{\mathrm{q}}$ can be taken as the gas density at the planet surface in the case of $r_{\mathrm{p}} \ll r_{\mathrm{B}}$, and is given by

$$
\begin{aligned}
\rho_{\mathrm{q}}= & 4.6 \times 10^{-6}\left(\frac{\kappa}{0.1 \mathrm{~m}^{2} \mathrm{~kg}^{-1}}\right)\left(\frac{\tilde{\tau}_{\text {grow }, \mathrm{p}}}{6.0 \times 10^{5} \text { years }}\right) \\
& \cdot\left(\frac{m_{\mathrm{p}}}{1.0}\right)^{7 / 3} \mathrm{~g} \mathrm{~cm}^{-3},
\end{aligned}
$$

where $\kappa$ is the opacity.

Next we consider the subdisk. Considering the case that gas with large angular momentum has steadily supplied from the nebula, not an atmosphere but a subdisk can be formed inside of the Bondi radius of a planet as large as the Earth (Miki, 1982; Korycansky and Papaloizou, 1996). In this case the boundary of the planetary atmosphere and the subdisk is determined as the processes of angular momentum transfer, which is yet an open question. The structure of the subdisk is uncertain, so that we here adopt a nominal model with a single power-law density distribution:

$$
\rho_{\mathrm{g}, \mathrm{D}}\left(a_{\mathrm{s}}\right)=\rho_{\mathrm{g} 0}\left(\frac{a_{\mathrm{s}}}{r_{\mathrm{B}}}\right)^{-n} \quad\left(\text { for } a_{\mathrm{s}} \leq r_{\mathrm{B}}\right),
$$

where $n$ is the power-law index. We also assume that the subdisk is isothermal. Then $\eta$ in Eq. (45) is given by

$$
\begin{aligned}
\eta\left(a_{\mathrm{s}}\right)=1-\left(1-n \frac{a_{\mathrm{s}}}{r_{\mathrm{B}}}\right)^{1 / 2} & \simeq \frac{n}{2} \frac{a_{\mathrm{s}}}{r_{\mathrm{B}}} \\
& \left(\text { for } a_{\mathrm{s}} \ll r_{\mathrm{B}}\right) .
\end{aligned}
$$

Adopting $m_{\mathrm{p}}=1.0, \mu_{\mathrm{s}}=\mu_{\mathrm{M}}$, and $\tilde{\tau}_{\text {grow }, \mathrm{p}}=6.0 \times 10^{5}$ years (see Subsection 4.1) we calculate $\tau_{\text {gas }}$ from Eq. (45) for the two cases; $\tau_{\text {gasA }}$ for the hydrostatic atmosphere and $\tau_{\text {gasD }}$ for the subdisk with $n=1.5$. The results are shown in Fig. 11. For comparison we also show the tidal evolution time $\tau_{\text {tidal }}$ of $a_{\mathrm{s}}$ for $Q_{\mathrm{p}}=400$ and the orbital decay time $\tau_{\text {acc }}$ due to accretion drag. We find that $\tau_{\text {gasA }}$ is shorter than both $\tau_{\text {acc }}$ and $\tau_{\text {tidal }}$ for all $a_{\mathrm{s}}$. This means, for the case of static atmosphere, gas drag is the main agent of the orbital decay and satellite inevitably spirals into the planet. On the other hand, $\tau_{\text {gasD }}$ is somewhat longer than $\tau_{\text {acc }}$ with $n=1.5$, so that evolution of $a_{\mathrm{s}}$ is determined mainly by the balance between accretion drag and tidal repulsion. Even in the case of smaller $m_{\mathrm{p}}$ or $\mu_{\mathrm{s}}, \tau_{\mathrm{gasD}}$ is still longer than $\tau_{\mathrm{acc}}$, so that 


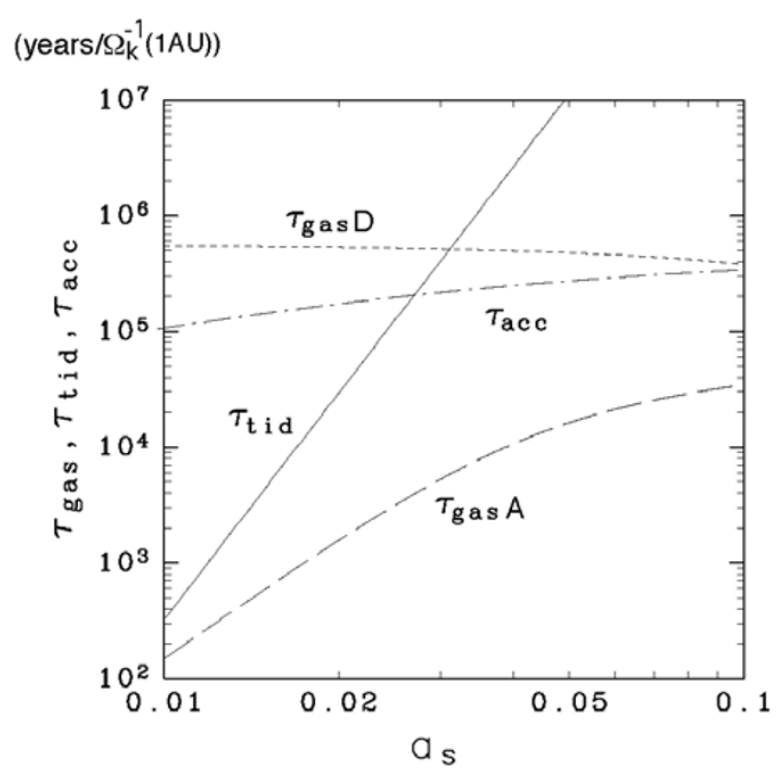

Fig. 11. Orbital decay time scales of the satellite by gas drag ( $\tau_{\text {gasA }}$ for the hydrostatic atmosphere and $\tau_{\text {gasD }}$ for the subdisk) in the solar nebular as functions of $a_{\mathrm{s}}$ with $m_{\mathrm{p}}=1.0$ and $\mu_{\mathrm{s}}=\mu_{\mathrm{M}}$. We adopt $n=1.5$ to $\tau_{\text {gasD }}, \tilde{\tau}_{\text {grow,E }}=6 \times 10^{5}$ years to $\tau_{\text {gasA }}$ and $\tau_{\text {acc }}$, and $Q_{\mathrm{p}}=400$ to $\tau_{\text {tid }}$, respectively.

the picture of co-accretion shown in Subsection 4.1 might not be affected by the effect of gas drag. It should be noted that even in such cases the protoatmosphere must dissipate immediately after the completion of planetary growth.

Since the lunar mass is comparable to or larger than that of the protoatmosphere of the Earth, the Moon would affect the structure and evolution of the atmosphere. The analysis of the coupled system is desirable.

\subsection{Stochastic impacts}

Until now, we only considered the "ordered component" of accumulated angular momentum, i.e., angular momentum brought by a large number of small planetesimals. However, the "stochastic component" of accumulated angular momentum, i.e., angular momentum brought by one or a few number of large planetesimals to the planet (or the satellite) may play a significant role, in particular, in co-accretion after the giant impact (Subsection 4.2). Here we estimate which component is dominant in the evolution of the total and the orbital angular momentums of the Earth-Moon system after the giant impact.

The $z$-component of mean-square angular momentum supplied to a planet-satellite system during co-accretion is given by (e.g., Dones and Tremaine, 1993b)

$$
\begin{aligned}
\left\langle L_{z}^{2}\right\rangle= & {\left[M_{\mathrm{r}, \mathrm{p}}\left\langle l_{z}\right\rangle_{\mathrm{p}}+M_{\mathrm{r}, \mathrm{s}}\left\langle l_{z}\right\rangle_{\mathrm{s}}\right]^{2} } \\
& +\left[m_{\mathrm{q}, \mathrm{p}}^{2}\left\langle l_{z}^{2}\right\rangle_{\mathrm{p}}+m_{\mathrm{q}, \mathrm{s}}^{2}\left\langle l_{z}^{2}\right\rangle_{\mathrm{s}}\right],
\end{aligned}
$$

with

$$
m_{\mathrm{q}, k}^{2}=\frac{\left\langle m^{2}\right\rangle_{k} M_{\mathrm{r}, k}}{\langle m\rangle_{k}}
$$

where $M_{\mathrm{r}, k},\left\langle l_{z}\right\rangle_{k},\left\langle l_{z}^{2}\right\rangle_{k},\langle m\rangle_{k}$, and $\left\langle m^{2}\right\rangle_{k}$ are the total mass, the mean and mean-square angular momentums per unit mass (which are the dimensional angular momentums: $l_{z}=$ $\left.\ell_{z} R_{\mathrm{H}}^{2} \Omega_{\mathrm{k}}\right)$, and the mean and mean-square masses of planetesimals, respectively, colliding with the planet $(k=\mathrm{p})$ or with the satellite $(k=\mathrm{s})$. Further, $m_{\mathrm{q}, k}^{2}$ denotes the product of the effective mass $\left(\left\langle m^{2}\right\rangle_{k} /\langle m\rangle_{k}\right)$ and the total mass of planetesimals. The bracketed first and second terms in the right hand side of Eq. (51) correspond to the ordered and the stochastic components, respectively. Similarly, if we consider the evolution of the orbital angular momentum of the satellite, the $z$-component of mean-square angular momentum supplied to a satellite is given by

$$
\left\langle L_{z}^{2}\right\rangle_{\mathrm{s}}=M_{\mathrm{r}, \mathrm{s}}^{2}\left\langle l_{z}\right\rangle_{\mathrm{s}}^{2}+m_{\mathrm{q}, \mathrm{s}}^{2}\left\langle l_{z}^{2}\right\rangle_{\mathrm{s}} .
$$

We first evaluate the ordered component of Eqs. (51) and (53). In Subsection 4.2, we showed that the total angular momentum of a planet-satellite system is decreased by anisotropic collisions of planetesimals to the satellite and the escaping ejecta. According to Fig. 5 and the calculation of the ejecta orbits (see Subsection 5.2), we obtain

$$
\begin{aligned}
\left|M_{\mathrm{r}, \mathrm{s}}\left\langle l_{z}\right\rangle_{\mathrm{s}}\right| & =\left[0.2+1.0\left(1-P_{\mathrm{co}}\right)\right]\left\langle l_{o}\right\rangle M_{\mathrm{r}, \mathrm{s}} \\
& \simeq\left[0.1+0.7\left(1-P_{\mathrm{co}}\right)\right] \frac{L_{\mathrm{EM}}}{M_{\mathrm{E}}} M_{\mathrm{r}, \mathrm{p}},
\end{aligned}
$$

where $\left\langle l_{o}\right\rangle$ is the mean specific orbital angular momentum of the satellite and the lower right hand side comes from the results shown in Fig. 9(a). The range of mean specific angular momentum of planetesimals accumulating onto the planet is given by $\left|\left\langle l_{z}\right\rangle_{\mathrm{p}}\right| \leq 0.05 L_{\mathrm{EM}} / M_{\mathrm{E}}$ (Dones and Tremaine, 1993a) when the spatial distribution of planetesimals in the circumsolar disk is uniform and the relative-random velocities are as large as that adopted in Subsection 4.2. Thus we can safely neglect the contribution of planetary accretion to the ordered component in Eq. (51).

Next we evaluate the stochastic component of Eqs. (51) and (53). The value of $m_{\mathrm{q}, k}$ is determined by the mass distribution of planetesimals. We consider that numbers of planetesimals per unit mass are given by a power-low as $n_{k}(m) \propto m^{-q}\left(m_{\min , k}<m<m_{\max , k}\right.$, where $k$ denotes the subscripts "p" and "s"). Here we assume that $m_{\max , k}$ is defined as

$$
\int_{m_{\max , k}}^{\infty} n_{k} d m=1 .
$$

Then, we obtain that

$$
m_{\mathrm{q}, k}^{2}=\left\{\begin{array}{l}
\frac{(2-q)^{2}}{(3-q)(q-1)} M_{\mathrm{r}, k}^{2} \\
\quad(\text { for } 1<q<2), \\
\frac{(q-2)^{2}}{(3-q)(q-1)}\left(\frac{m_{\min , k}}{m_{\max , k}}\right)^{2(q-2)} M_{\mathrm{r}, k}^{2} \\
(\text { for } 2<q<3) .
\end{array}\right.
$$

For the case of $1<q<2$, most of the mass of the impactors is contained in the largest planetesimals, so that $m_{\mathrm{q}, k}$ is comparable with $M_{\mathrm{r}, k}$. On the other hand, for the case of $2<q<3, m_{\mathrm{q}, k}$ is much smaller than $M_{\mathrm{r}, k}$ since most of the mass of the impactors is concentrated in the smallest planetesimals.

Since the square root of the specific mean-square angular momentums of planetesimals colliding with the satellite is 
almost same as that of the satellite, the stochastic components about the satellite is approximately given by

$$
m_{\mathrm{q}, \mathrm{s}}\left\langle l_{z}^{2}\right\rangle_{\mathrm{s}}^{1 / 2} \simeq m_{\mathrm{q}, \mathrm{s}}\left\langle l_{o}\right\rangle
$$

From Eq. (56) and Fig. 9(d), we find that $m_{\mathrm{q}, \mathrm{s}}^{2} / m_{\mathrm{q}, \mathrm{p}}^{2} \sim$ $\left(M_{\mathrm{s}} / M_{\mathrm{p}}\right)^{2}\left(\ll\left\langle l_{z}^{2}\right\rangle_{\mathrm{p}} /\left\langle l_{z}^{2}\right\rangle_{\mathrm{s}} \sim R_{\mathrm{p}} / A_{\mathrm{s}}\right)$. Thus, we can neglect the effect of the stochastic impact with the satellite relative to that with the planet in Eq. (51). Hence, we focus on the stochastic component about the planet for the evaluation of the evolution of the total angular momentum. Taking the mean impact velocity to be the escape velocity of the planet and the mean impact parameter to be $R_{\mathrm{p}} / \sqrt{2}$, we obtain

$$
\begin{aligned}
& m_{\mathrm{q}, \mathrm{p}}\left\langle l_{z}^{2}\right\rangle_{\mathrm{p}}^{1 / 2} \\
& =\left\{\begin{array}{c}
\frac{8.6(2-q) m_{\mathrm{p}}^{2 / 3}}{(3-q)^{1 / 2}(q-1)^{1 / 2}} M_{\mathrm{r}, \mathrm{p}} \frac{L_{\mathrm{EM}}}{M_{\mathrm{E}}} \\
(\text { for } 1<q<2), \\
\frac{8.6(2-q) m_{\mathrm{p}}^{2 / 3}}{(3-q)^{1 / 2}(q-1)^{1 / 2}} M_{\mathrm{r}, \mathrm{p}}\left(\frac{m_{\min , \mathrm{p}}}{m_{\max , \mathrm{p}}}\right)^{q-2} \frac{L_{\mathrm{EM}}}{M_{\mathrm{E}}} \\
(\text { for } 2<q<3),
\end{array}\right.
\end{aligned}
$$

where we should note that $m_{\mathrm{p}}$ is the normalized mass of the planet by the Earth mass.

Now we compare the ordered components (Eq. (54)) with the stochastic components (Eqs. (57) and (58)). For the case of $1<q<2$, the stochastic component about $\left\langle L_{z}^{2}\right\rangle$ is much larger than the ordered component and the stochastic component about $\left\langle L_{z}^{2}\right\rangle_{\mathrm{s}}$ is comparable or slightly larger than the ordered component. On the other hand, for the case of $2<q<3$, the stochastic components are negligible. Thus, evolution of the angular momentums of the planet-satellite system are determined by the ordered components if $q>2$.

Then we discuss the value of $q$ in the final stage of planetary accretion. In general, we must consider the possibility that collisions of the protoplanets (i.e., runaway bodies, which are detached from the continuous power-law mass distribution (e.g., Kokubo and Ida, 1996)) determine the stochastic components, since the Moon-forming giant impact itself is one of the example. In the following discussion, however, we assume that there was no impact of other protoplanets with the proto-Earth after the Moon-forming impact. If other protoplanets exist around the proto-Earth in the final stage of its accretion, they affect the heliocentric orbit of the proto-Earth and the present small orbital eccentricity of the Earth would not be achieved whereas dynamical friction of small planetesimals might decrease the eccentricity of the proto-Earth (Agnor et al., 1999). Thus, we focus on the value of $q$ only for planetesimals except runaway bodies. In the stage of runaway growth, $q$ is shown to be $\sim 2.5$ (Kokubo and Ida, 1996). The value of $q$ might become smaller through collisional disruption, since $q \sim 11 / 6$ for steady state of the collision cascade (Dohnanyi, 1969; Tanaka et al., 1996). The disruption is, however, expected to be less effective for larger planetesimals with stronger gravity. In fact, statistical simulations by Wetherill and Stewart (1993) showed that the value of $q$ for small fragments $\left(\lesssim 10^{18} \mathrm{~g}\right)$ is $\sim 11 / 6$, whereas that for residual planetesimals $\left(10^{18} \mathrm{~g} \lesssim m \lesssim 10^{24} \mathrm{~g}\right)$ is $\sim 2.5$. This means that most of the mass of the impactors is contained in the small planetesimals $\left(\sim 10^{18} \mathrm{~g}\right)$. Thus, we con- sider that the stochastic components would not be dominant in co-accretion after the giant impact.

\section{Summary}

Based on the orbital calculations of Keplerian planetesimals, we develop a numerical model which describes accretional and dynamical evolution of planet-satellite systems in a swarm of planetesimals with various spatial and velocity distributions.

Obtained mass accretion rate of the satellite is in good agreement with the particle-in-a-box model by Harris and Kaula (1975) in the case of uniform spatial distribution of planetesimals with $\sqrt{e^{2}+i^{2}} \gtrsim 4$.0. In the cases of nonuniform spatial distributions, however, the absolute values of mass accretion rate in the particle-in-a-box model is no longer valid. Even in these cases, the ratio of mass accretion rate of the satellite to that of the planet is still in good agreement with an analytic solution derived from particle-in-a-box approximation.

Mean specific angular momentum supplied to the satellite $J_{\mathrm{s}}$ from planetesimals with uniform spatial distribution is always negative, even for the case of large velocity dispersion. This is because incident of planetesimals is not isotropic for the satellite due to its prograde motion around the planet. But the values of $J_{\mathrm{s}}$ become positive for the case of nonuniform spatial distribution with small $E_{\max }$. These results are consistent with the results of the previous work about planetary spin by accretion of planetesimals (Ohtsuki and Ida, 1998).

Using these results, we calculate evolution of planet-satellite systems. In the plane of orbital radius of the satellite vs. satellite/planet mass ratio, a satellite moves quickly toward the balanced orbital radius where accretion drag compensates with tidal repulsion and then grows slowly toward the equilibrium satellite/planet mass ratio. Analyses of the model show that, as long as from the dynamical viewpoint, two types of co-accretion scenarios for the origin of the Moon are plausible as follows:

(1) In the first scenario, the Moon started from a small embryo with its mass of $10^{-2} M_{\mathrm{M}}$ and grew in the swarm of planetesimals with low velocity dispersion and nonuniform spatial distribution, so that large angular momentum was supplied to the planetary spin. Such situations would be realized when the Earth grew rapidly before the dissipation of the solar-nebula. The rapid growth of the Earth ( $\tilde{\tau}_{\text {grow,E }} \sim 10^{6}$ years) was needed for the semimajor axis of the Moon to be kept so small that the Moon grew up more rapidly than the Earth under the benefit of large gravitational focusing by the Earth. Moreover, the rapid growth prevented the Moon from spiraling into the Earth by gas drag.

(2) In the second scenario, the Moon was formed by a giant impact occurred during Earth accretion. The impact supplied enough angular momentum as large as that of the present Earth-Moon system. Since a smaller planet cannot have such large angular momentum, mass of the proto-Earth immediately after the giant impact should be larger than about half of the present Earth mass. The Earth grew rather slowly in the vacuum $\left(\tilde{\tau}_{\text {grow,E }} \sim\right.$ $10^{8}$ years) due to large velocity dispersion of planetesi- 
mals. The dynamical evolution of the Earth-Moon system strongly depended on coalescence probability between the Moon and planetesimals. For the perfect accretion case, the mass ratio of the Moon hardly changed, whereas the total angular momentum decreased a little $(\sim 5 \%)$ due to anisotropic collisions of planetesimals to the Moon. On the other hand, if the Moon did not grow, much of the angular momentum ( 25\%) was taken away by escaping impact ejecta. From a comparison with results of giant-impact simulations, we found that the Moon-forming giant impact occurred when the total mass of the impactor and the proto-Earth was 0.7 times of the present Earth mass.

Acknowledgments. We are grateful to K. Ohtsuki and R. M. Canup for giving valuable comments and suggestions as reviewers. We also thank H. Tanaka and T. Tanigawa for helpful comments. The figures were produced by the GFD-DENNOU Library.

\section{Appendix A. Upper Limit of the Enlargement Fac- tor $g_{3}$}

Here we briefly explain the derivation of the upper limit of the enlargement factor of the satellite's cross section $g_{3}$ for oblique penetrations of planetesimals given in Eq. (13). In the limit of $i \rightarrow 0,\left\langle 1 / \cos \theta_{3}\right\rangle$ diverses, whereas the collision probability of a satellite with penetrating planeteismals through an anulus (which contains the satellite) should remain finite, so that $g_{3}$ should be confined by some upper limit.

We determine the upper limit by the condition that the value of the collision rate $P_{\mathrm{s}}(e, i)$ must converge to the value for the two-dimensional case when $i \rightarrow 0$. From Eqs. (10), (12), and (14), we obtain the following condition

$$
\lim _{i \rightarrow 0} \frac{\left\langle g_{3}\right\rangle r_{\mathrm{s}}^{2}}{2 a_{\mathrm{s}} \Delta a} n_{3}=\frac{\left\langle g_{2}\right\rangle r_{\mathrm{s}}}{\pi a_{\mathrm{s}}} n_{2}
$$

where $\left\langle g_{3}\right\rangle$ and $\left\langle g_{2}\right\rangle$ are average values of $g_{3}$ and $g_{2}$, respectively, $n_{3}$ and $n_{2}$ denote numbers of penetrating orbits through an anulus and crossing orbits with the satellite orbit, respectively.

Then we estimate the ratio of $n_{3}$ to $n_{2}$. We find, from numerical calculations, that number of planetesimals with the closest distance $r_{\min }$ from the planet is proportional to $r_{\text {min }}^{\beta}$ with $\beta \sim 0$ for smaller $e(\lesssim 4)$. Further, assuming that orbits of planetesimals are parabolic, we obtain $n_{3} / n_{2}=\Delta a /\left(2 a_{\mathrm{s}}\right)$. Hence, we obtain the upper limit of $\left\langle g_{3}\right\rangle$ as

$$
\left\langle g_{3}\right\rangle=\frac{4\left\langle g_{2}\right\rangle}{\pi} \frac{a_{\mathrm{s}}}{r_{\mathrm{s}}} \sim 3 \frac{a_{\mathrm{s}}}{r_{\mathrm{s}}},
$$

where the factor 3 in the right hand side is determined by numerical fitting. Note that this factor is somewhat nominal, since it really depends on $e$ and $a_{\mathrm{s}}$.

\section{Appendix B. Behavior of $\boldsymbol{P}_{\mathrm{S}}$ and $\boldsymbol{J}_{\mathrm{S}}$ in the Case of $\boldsymbol{e}=\boldsymbol{i}=\mathbf{0}$}

We here derive approximate form of the collision rate $P_{\mathrm{s}}$ and the mean specific planetocentric angular momentum $J_{\mathrm{s}}$ as functions of the satellite's orbital radius $a_{\mathrm{s}}$ from the minimum distance $r_{\text {min }}$ between a planetesimal (with orbital elements

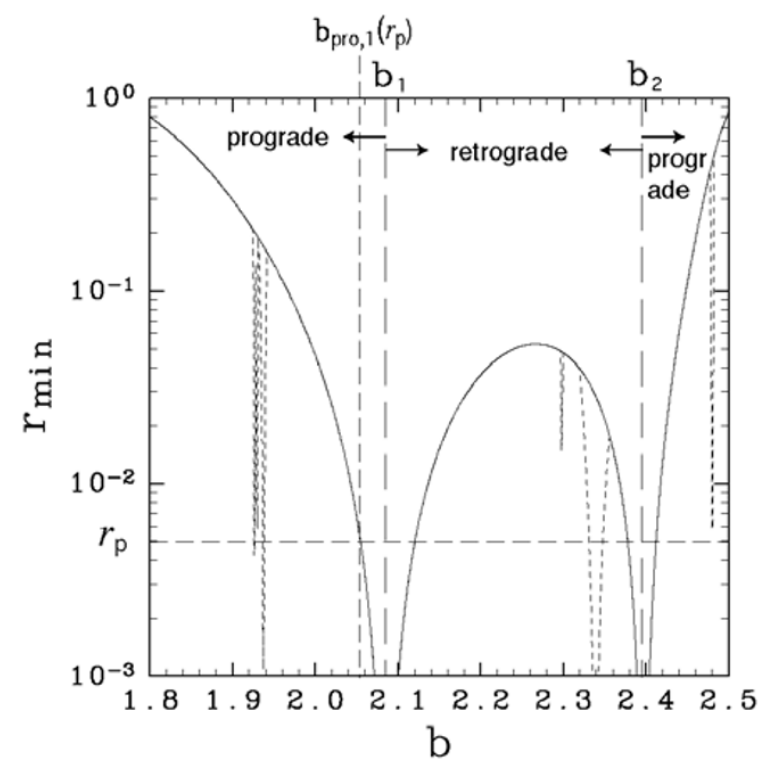

Fig. 12. Minimum distance $r_{\text {min }}$ between the planet and a planetesimal for the first encounter (solid curve) and including multi-time encounters (dashed curve) in the case of $e=i=0$. Radius of the planet $r_{\mathrm{p}}$ is shown by a horizontal dashed line. Direction of rotation of the planetesimal around the planet is changed at $b_{1}$ and $b_{2}$.

$b, e$, and $i$ ) and the planet during the encounter. We focus on the case of $e=i=0$. Figure 12 shows $r_{\min }$ as a function of $b$. There are two main collision bands (where $r_{\min }<a_{\mathrm{s}}$ ) around $b=b_{1} \sim 2.1$ and $b=b_{2} \sim 2.4$. Planetesimals are prograde around the planet in the ranges of $b<b_{1}$ and $b>b_{2}$, and retrograde in the range of $b_{1}<b<b_{2}$.

From Eqs. (10) and (16), $P_{\mathrm{s}}(e=i=0)$ is approximately described by

$$
\begin{aligned}
& P_{\mathrm{s}}\left(a_{\mathrm{s}}, r_{\mathrm{s}}\right) \\
& =\frac{3 r_{\mathrm{s}}}{2 \pi a_{\mathrm{s}}}\left[\left\langle\frac{1}{\sin \theta_{2}}\right\rangle_{\text {pro }}\left(2 S_{\text {pro }}\left(a_{\mathrm{s}}\right)-S_{\text {pro }}\left(r_{\mathrm{p}}\right)\right)\right. \\
& \left.+\left\langle\frac{1}{\sin \theta_{2}}\right\rangle_{\text {retro }}\left(2 S_{\text {retro }}\left(a_{\mathrm{s}}\right)-S_{\text {retro }}\left(r_{\mathrm{p}}\right)\right)\right],
\end{aligned}
$$

where $\left\langle 1 / \sin \theta_{2}\right\rangle_{\text {pro }},\left\langle 1 / \sin \theta_{2}\right\rangle_{\text {retro }}$ are averaged inverses of $\sin \theta_{2}$ for prograde and retrograde orbits, which are estimated as about 1.1 and 2.2, respectively, by numerical calculations. In the above, $S_{\text {pro }}$ and $S_{\text {retro }}$ are defined by

$$
S_{k}(r)=\sum_{j=1}^{2}\left|b_{j}^{2}-b_{k, j}(r)^{2}\right|,
$$

where subscript $k$ denotes "pro" (or "retro"), and $b_{k, j}(r)$ are the differential semimajor axes of the orbits with $r_{\min }=r$ in the prograde (or retrograde) range beside $b_{j}$. The factors of $S_{k}$ in Eq. (B.1) represent the fact that the planetesimal crosses each satellite orbit with $a_{\mathrm{s}}>r_{\text {min }}$ twice if $r_{\min }>r_{\mathrm{p}}$ and once if $r_{\min }<r_{\mathrm{p}}$.

From Fig. 12 we find

$$
\begin{gathered}
S_{\text {pro }}(r) \simeq 2.7 r^{1 / 2}, \\
S_{\text {retro }}(r) \simeq \begin{cases}5.5 r^{1 / 2} & (r \leq 0.06), \\
b_{2}^{2}-b_{1}^{2} & (r>0.06) .\end{cases}
\end{gathered}
$$


It should be noticed that there are no retrograde orbits with $r_{\min }>0.06$.

Substituting Eqs. (B.3) and (B.4) into Eq. (B.1), we obtain

$$
P_{\mathrm{s}}\left(a_{\mathrm{s}}, r_{\mathrm{s}}\right) \simeq\left\{\begin{array}{c}
14.4 r_{\mathrm{s}}\left(a_{\mathrm{s}}^{1 / 2}-0.035\right) a_{\mathrm{s}}^{-1} \\
\left(a_{\mathrm{s}} \leq 0.06\right), \\
2.84 r_{\mathrm{s}}\left(a_{\mathrm{s}}^{1 / 2}+0.82\right) a_{\mathrm{s}}^{-1} \\
\left(a_{\mathrm{s}}>0.06\right) .
\end{array}\right.
$$

The approximate formula well coincides with numerical results. This functional change of $P_{\mathrm{s}}(e=i=0)$ at $a_{\mathrm{s}} \sim 0.06$ is appeared as a kink in Fig. 3.

Similarly, $J_{\mathrm{s}}(e=i=0)$ is obtained approximately by

$$
J_{\mathrm{S}}\left(a_{\mathrm{s}}\right)=\frac{\sum_{k=\text { pro,retro }}\left\langle\frac{1}{\sin \theta_{2}}\right\rangle_{k}\left(2 S_{k}\left(a_{\mathrm{s}}\right)-S_{k}\left(r_{\mathrm{p}}\right)\right)\left\langle\frac{\ell_{z}}{\ell_{\mathrm{o}}}\right\rangle_{k}}{\sum_{k=\text { pro, retro }}\left\langle\frac{1}{\sin \theta_{2}}\right\rangle_{k}\left(2 S_{k}\left(a_{\mathrm{s}}\right)-S_{k}\left(r_{\mathrm{p}}\right)\right)} .
$$

Here, approximate mean values of specific angular momentums are $\left\langle\ell_{z} / \ell_{\mathrm{o}}\right\rangle_{\text {pro }} \sim 1$ for prograde orbits and $\left\langle\ell_{z} / \ell_{\mathrm{o}}\right\rangle_{\text {retro }} \sim$ -1 for retrograde orbits, respectively. Substituting Eqs. (B.3) and (B.4) into Eq. (B.6), we obtain

$$
J_{\mathrm{s}}\left(a_{\mathrm{s}}\right)=\left\{\begin{array}{c}
-0.61 \\
\left(a_{\mathrm{s}} \leq 0.06\right), \\
\left(a_{\mathrm{s}}^{1 / 2}-0.89\right) /\left(a_{\mathrm{s}}^{1 / 2}+0.82\right) \\
\left(a_{\mathrm{s}} \geq 0.06\right) .
\end{array}\right.
$$

This is roughly coincide with numerical results. For $a_{\mathrm{s}} \geq$ 0.06 , number of collisional planetesimals with prograde orbits around the planet increases with $a_{\mathrm{s}}$, wheras number of retrograde orbits is kept to be a constant, so that $J_{\mathrm{s}}$ increases with $a_{\mathrm{s}}$ (see Fig. 5).

\section{References}

Abe, Y. and T. Matsui, The formation of an impact generated atmosphere and its implication for the early thermal history of the Earth, J. Geophys. Res., 90, 545-559, 1985.

Abe, Y. and T. Matsui, Early evolution of the Earth: Accretion, atmosphere formation, and thermal history, J. Geophys. Res., 91, 291-302, 1986.

Adachi, I., C. Hayashi, and K. Nakazawa, The gas drag effect on the elliptic motion of a solid body in the primordial solar nebula, Prog. Theor. Phys., 56, 1756-1771, 1976.

Agnor, C. B., R. M. Canup, and H. F. Levison, On the character and consequences of large impacts in the late stage of terrestrial planet formation, Icarus, 142, 219-237, 1999.

Benz, W., W. L. Slattery, and A. G. W. Cameron, The origin of the Moon and the single impact hypothesis I, Icarus, 66, 515-535, 1986

Benz, W., W. L. Slattery, and A. G. W. Cameron, The origin of the Moon and the single impact hypothesis II, Icarus, 71, 30-45, 1987.

Benz, W., A. G. W. Cameron, and H. J. Melosh, The origin of the Moon and the single impact hypothesis III, Icarus, 81, 113-131, 1989.

Boss, A. P. and S. J. Peale, Dynamical constraints on the origin of the Moon, in Origin of the Moon, edited by W. K. Hartmann, R. J. Phillips, and G. J. Taylor, 59 pp., Lunar and planetary Institute, Houston, 1986.

Burns, J. A., Some back ground about satellites, in Satellites, edited by J. A. Burns and M. S. Matthews, 1 pp., Univ. Arizona Press, Tucson, 1986.

Cameron, A. G. W, The origin of the Moon and the single impact hypothesis V, Icarus, 126, 126-137, 1997.

Cameron, A. G. W. and W. Benz, The origin of the Moon and the single impact hypothesis IV, Icarus, 92, 204-216, 1991.

Cameron, A. G. W. and R. M. Canup, The giant impact occurred during Earth accretion, 29th Lunar and Planet. Sci., abstract 1062 pp., 1998a.
Cameron, A. G. W. and R. M. Canup, The giant impact and the formation of the Moon, Origin of the Earth and Moon, abstract, 3 pp., 1998b.

Canup, R. M. and L. W. Esposito, Accretion of the Moon from an impactgenerated disk, Icarus, 119, 427-446, 1996.

Canup, R. M., W. R. Ward, and A. G. W. Cameron, A scaling relationship for satellite-forming impacts, Icarus, 2001 (submitted).

Chambers, J. E. and G. W. Wetherill, Making terrestrial planets: $N$-body integrations of planetary embryos in three dimensions, Icarus, 136, 304327, 1998.

Dohnanyi, J. S., Collisional model of asteroids and their debris, J. Geophys. Res., 74, 2531-2554, 1969.

Dones, L. and S. Tremaine, On the origin of planetary spins, Icarus, 103, 67-92, 1993a.

Dones, L. and S. Tremaine, Why does the Earth spin forward?, Science, 259, 350-354, 1993b.

Durisen, R. H. and R. A. Gingold, Numerical simulation of fission, in Origin of the Moon, edited by W. K. Hartmann, R. J. Phillips, and G. J. Taylor, 487 pp., Lunar and planetary Institute, Houston, 1986.

Gladman, B. J., J. A. Burns, M. J. Duncan, and H. F. Levison, The dynamical evolution of lunar impact ejecta, Icarus, 118, 302-321, 1995.

Goldreich, P. and S. Soter, Q in the solar system, Icarus, 5, 375-389, 1966

Greenzweig, Y. and J. J. Lissauer, Accretion rate of protoplanets, Icarus, 87, 40-77, 1990.

Harris, A. W., Satellite formation, II, Icarus, 34, 128-145, 1978.

Harris, A. W. and W. M. Kaula, A co-accretional model of satellite formation, Icarus, 24, 516-524, 1975 (HK75).

Hayashi, C., K. Nakazawa, and Y. Nakagawa, Formation of the solar system, in Protostars and Planets II, edited by D. C. Black and M. S. Matthews, 1100 pp., Univ. Arizona Press, Tucson, 1985.

Herbert, F., D. R. Davis, and S. J. Weidenschillig, Formation and evolution of a circumterestrial disk: Constraints on the origin of the Moon in geocentric orbit, in Origin of the Moon, edited by W. K. Hartmann, R. J. Phillips, and G. J. Taylor, 701 pp., Lunar and planetary Institute, Houston, 1986.

Holsapple, K. A., The scaling of impact processes in planetary sciences, Ann. Rev. Earth Planet. Sci., 21, 333-373, 1993.

Housen, K. R., R. M. Schmidt, and K. A. Holsapple, Crater ejecta scaling laws: Fundamental forms based on dimensional analysis, J. Geophys Res., 88, 2485-2499, 1983.

Ida, S., Stirring and dynamical friction rates of planetesimals in the solar gravitational field, Icarus, 88, 129-145, 1990.

Ida, S. and J. Makino, Scattering of planetesimals by a protoplanet: Slowing down of runaway growth, Icarus, 106, 210-227, 1993.

Ida, S. and K. Nakazawa, Collisional probability of planetesimals revolving in the solar gravitational field, III, Astron. Astrophys., 224, 303-315, 1989

Ida, S. and K. Nakazawa, Did rotation of the protoplanets originate from the successive collisions of planetesimals?, Icarus, 86, 561-573, 1990.

Ida, S., R. M. Canup, and G. R. Stewart, Lunar accretion from an impactgenerated disk, Nature, 389, 353-357, 1997.

Ito, T. and K. Tanikawa, Stability and instability of the terrestrial protoplanet system and their possible roles in the final stage of planet formation, Icarus, 139, 336-349, 1999.

Kaula, W. M., Thermal evolution of Earth and Moon growing by planetesimal impacts, J. Geophys. Res., 84, 999-1008, 1979.

Kokubo, E. and S. Ida, On runaway growth of planetesimals, Icarus, 123, 180-191, 1996.

Kokubo, E. and S. Ida, Oligarchic growth: Formation of proto-planets from planetesimals, Icarus, 131, 171-178, 1998.

Korycansky, D. G. and J. C. B. Papaloizou, A method for calculation of nonlinear shear flow: Application to formation of giant planets in the solar nebula, Astrophys. J. Suppl., 105, 181-190, 1996.

Lambeck, K., The Earth's Variable Rotation, 449 pp., Cambridge Univ. Press, 1980.

Lissauer, J. J. and D. M. Kary, The origin of systematic component of planetary rotation. I. planet on a circular orbit, Icarus, 94, 126-159, 1991.

Love, A. E. H., A Treatise on the Mathematical Theory of Elasticity, 643 pp., Cambridge Univ. Press, Cambridge, 1934.

Mignard, F., The evolution of the lunar orbit revisited. I, Moon and Planets, 20, 301-305, 1979.

Miki, S., The gaseous flow around a protoplanet in the primordial solar nebular, Prog. Theor. Phys., 67, 1053-1067, 1982.

Morishima, R. and S. Watanabe, Collision probabilities of planet/satellite systems with planetesimals and evolution of the mass ratio, Proc. 29th 
ISAS Lunar Planet. Symp., 135 pp., 1996.

Morishima, R. and S. Watanabe, Co-accretion of the Earth-Moon system after the Moon-forming giant impact, in preparation.

Nakazawa, K. and S. Ida, Hill's approximation in three body problem, Prog. Theor. Phys. Suppl., 96, 167-174, 1988.

Nakazawa, K., T. Komuro, and C. Hayashi, Origin of the Moon-Capture by gas drag of the Earth's primordial atmosphere, Moon and Planets, 28, 311-327, 1983.

Ohtsuki, K., Capture probability of colliding planetesimals: Dynamical constraints on accretion of planets, satellites and ring particles, Icarus, 106, 228-246, 1993

Ohtsuki, K. and S. Ida, Planetary rotation by accretion of planetesimals with nonuniform spatial distribution formed by the planet's gravitational perturbation, Icarus, 131, 393-420, 1998 (OI98).

Ray, R. Y., R. J. Eanes, and B. F. Chao, Detection of tidal dissipation in the solid Earth by satellite tracking and altimetry, Nature, 381, 595-597, 1996.

Ruskol, E. L., The origin of the Moon: Formation of a swarm of bodies around the Earth, Sov. Astron., 4, 657-668, 1960.

Sagan, C. and S. F. Dermott, The tides in the seas of Titan, Nature, 300, 731-733, 1982.

Sasaki, S. and K. Nakazawa, Metal-Silicate fractionation in the growing Earth; energy source for the terrestrial magma ocean, J. Geophys. Res, 91, 9231-9238, 1986.

Sasaki, S. and K. Nakazawa, Did a primary solar-type atmosphere exist around the proto-Earth?, Icarus, 85, 21-42, 1990.

Singer, S. F. and L. W. Bandermann, Where was the Moon formed?, Science, 170, 438-439, 1970 .

Stevenson, D. J., A. W. Harris, and J. I. Lunine, Origin of satellites, in Satellites, edited by J. A. Burns and M. S. Matthews, 39 pp., Univ. Arizona Press, Tucson, 1986.

Tanaka, H. and S. Ida, Distribution of planetesimals around a protoplanet in the nebular gas. II. Numerical simulations, Icarus, 125, 302-316, 1997.

Tanaka, H. and S. Ida, Growth of a migrating protoplanet, Icarus, 139, 350-366, 1999.

Tanaka, H., S. Inaba, and K. Nakazawa, Steady-state size distribution for self-similar collision cascade, Icarus, 123, 450-455, 1996.

Ward, W. R., Protoplanet migration by nebula tides, Icarus, 126, 261-281, 1997.

Wetherill, G. W. and G. R. Stewart, Formation of planetary embryos: Effects of fragmentation, low relative velocity, and independent variation of eccentricity and inclination, Icarus, 106, 190-209, 1993.

Wood, J. A., Moon over Mauna Loa: A review of hypotheses of formation of Earth's Moon, in Origin of the Moon, edited by W. K. Hartmann, R. J. Phillips, and G. J. Taylor, 17 pp., Lunar and planetary Institute, Houston, 1986.

R. Morishima (e-mail: ryuji@eps.nagoya-u.ac.jp) and S. Watanabe 